\title{
Glutamate Controls tPA Recycling by Astrocytes, Which in Turn Influences Glutamatergic Signals
}

\author{
Frédéric Cassé, ${ }^{1 \star}$ Isabelle Bardou, ${ }^{1 \star}$ Lydia Danglot,${ }^{2,3}$ Aurélien Briens, ${ }^{1}$ Axel Montagne, ${ }^{1}$ Jérôme Parcq, ${ }^{1}$ \\ Anuradha Alahari, ${ }^{1,4}$ Thierry Galli, ${ }^{2,3}$ Denis Vivien, ${ }^{1 *}$ and Fabian Docagne ${ }^{1 *}$ \\ ${ }^{1}$ Institut National de la Santé et de la Recherche Médicale, U919, Serine Proteases and Pathophysiology of the Neurovascular Unit, Université de Caen, \\ Groupement d'Internet Public Cyceron, 14073 Caen Cedex, France, ${ }^{2}$ Institut National de la Santé et de la Recherche Médicale, ERL U950, Membrane Traffic \\ in Neuronal and Epithelial Morphogenesis, Institut Jacques Monod, 75013 Paris, France, ${ }^{3}$ Centre National de la Recherche Scientifique UMR-7592, \\ University Denis Diderot/Paris 7, Institut Jacques Monod, 75013 Paris, France, and ${ }^{4}$ Accent Medical and Scientific Writing, 14000 Caen, France
}

Tissue-type plasminogen activator (tPA) regulates physiological processes in the brain, such as learning and memory, and plays a critical role in neuronal survival and neuroinflammation in pathological conditions. Here we demonstrate, by combining mouse in vitro and in vivo data, that tPA is an important element of the cross talk between neurons and astrocytes. The data show that tPA released by neurons is constitutively endocytosed by astrocytes via the low-density lipoprotein-related protein receptor, and is then exocytosed in a regulated manner. The exocytotic recycling of tPA by astrocytes is inhibited in the presence of extracellular glutamate. Kainate receptors of astrocytes act as sensors of extracellular glutamate and, via a signaling pathway involving protein kinase $\mathrm{C}$, modulate the exocytosis of tPA. Further, by thus capturing extracellular tPA, astrocytes serve to reduce NMDA-mediated responses potentiated by tPA. Overall, this work provides the first demonstration that the neuromodulator, tPA, may also be considered as a gliotransmitter.

\section{Introduction}

In the last two decades, a number of key advances in the understanding of the physiology of astrocytes demonstrated their role in modulating synaptic transmission, synapse formation and plasticity, integrity of the blood-brain barrier, neurotoxicity, and nervous system repair (Haydon and Carmignoto, 2006). All these functions are in part related to their ability to regulate the concentration of neurotransmitters and neuromodulators in the extracellular space. For instance, astrocytes were shown to uptake and/or release glutamate (Araque et al., 1998; 2000), GABA (Bender and Norenberget, 2000), D-serine (Mothet et al., 2005), and ATP (Coco et al., 2003). Astrocytic release of glutamate and ATP occurs in response to calcium rise through vesicular exocytosis mediated by soluble $N$-ethylmaleimidesensitive factor attachment protein receptor (SNARE) (Volterra and Meldolesi, 2005; Galli and Haucke, 2004). Although uptake and release of small molecules, termed gliotransmitters, from astrocytes,

Received Oct. 20, 2011; revised Jan. 16, 2012; accepted Feb. 22, 2012.

Author contributions: F.C., I.B., A.B., A.A., D.V., and F.D. designed research; F.C., I.B., L.D., A.B., A.M., and A.A. performed research; L.D., J.P., and T.G. contributed unpublished reagents/analytic tools; F.C., I.B., A.B., T.G., D.V., and F.D. analyzed data; F.C., L.D., A.A., T.G., D.V., and F.D. wrote the paper.

This work was supported by grants from the Institut National de la Santé et de la Recherche Médicale and the Conseil Régional de Basse-Normandie. F.C. is contracted through the framework of the European FP7 (Eurostroke Network). We thank Professor Dudley K. Strickland for providing us with the rabbit polyclonal anti-mouse LRP1, and Dr. C. Streamson for plasmid vector $p$ Synapsin.

*F.C, I.B, D.V., and F.D. contributed equally to this work.

Correspondence should be addressed to Fabian Docagne, PhD, Centre Cyceron, BP5229, 14074 Caen Cedex, France. E-mail: docagne@cyceron.fr.

I. Bardou's present address: The Ohio State University, Psychology Department, 1835 Neil Avenue, Psychology Building, Columbus, $\mathrm{OH} 43210$.

DOI:10.1523/JNEUROSCI.5296-11.2012

Copyright $\odot 2012$ the authors $\quad 0270-6474 / 12 / 325186-14 \$ 15.00 / 0$ are largely described, fewer data support trafficking of larger molecules, such as proteins.

Tissue-type plasminogen activator (tPA) belongs to the family of serine proteases. Historically, tPA was first shown to be produced and released in circulation by endothelial cells, where it plays critical roles in inflammation, clotting, and fibrinolysis.

In the brain parenchyma, tPA is widely expressed in neurons and astrocytes. It is involved in cell migration, neuronal plasticity, integrity of the blood-brain barrier, inflammation, and proneurotoxicity (Tsirka et al., 1997; Baranes et al., 1998; Wang et al., 1998; Nicole et al., 2001). When released into the extracellular space, tPA mediates generation of plasmin, which regulates the degradation of the extracellular matrix (Tsirka et al., 1997) and the conversion of growth-factor and chemokine precursors into their active forms (Sheehan et al., 2007, Su et al., 2008).

Additional substrates, binding proteins, or receptors for tPA include the NMDAR, the low-density lipoprotein receptorrelated protein (LRP), and annexin-II (Siao and Tsirka, 2002; Samson et al., 2008; Zhang et al., 2009). For instance, tPA regulates NMDAR signaling, leading to neuronal cell death in pathological conditions, such as stroke (Nicole et al., 2001).

In view of all such functions of tPA in the CNS, this serine protease is now referred to as a neuromodulator (Fernández-Monreal et al., 2004; Samsom and Medcalf, 2006). Consequently, mechanisms that govern the availability of tPA in the extracellular space strongly influence tPA-modulated processes. The present study examines whether tPA acts as a gliotransmitter and, in particular, whether astrocytes are able to control the amount of tPA in the extracellular compartment and, if so, by which mechanism.

The results indicate that (1) astrocytes rapidly take up neuron-derived tPA through constitutive LRP-mediated, 
A

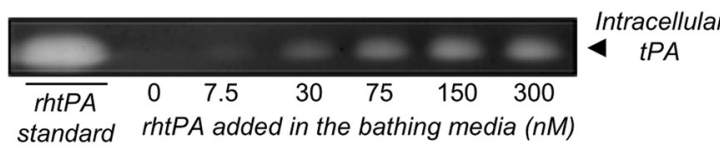

D
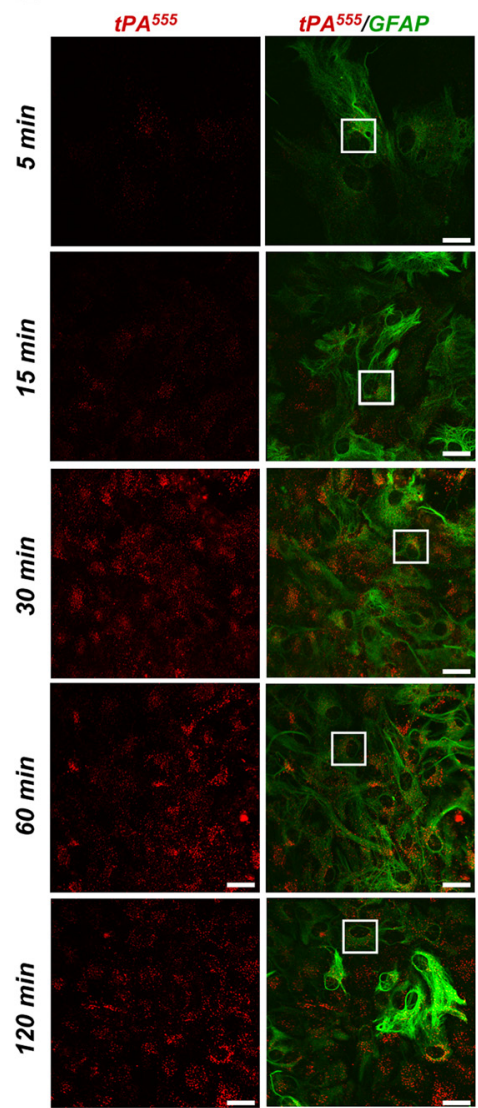

B
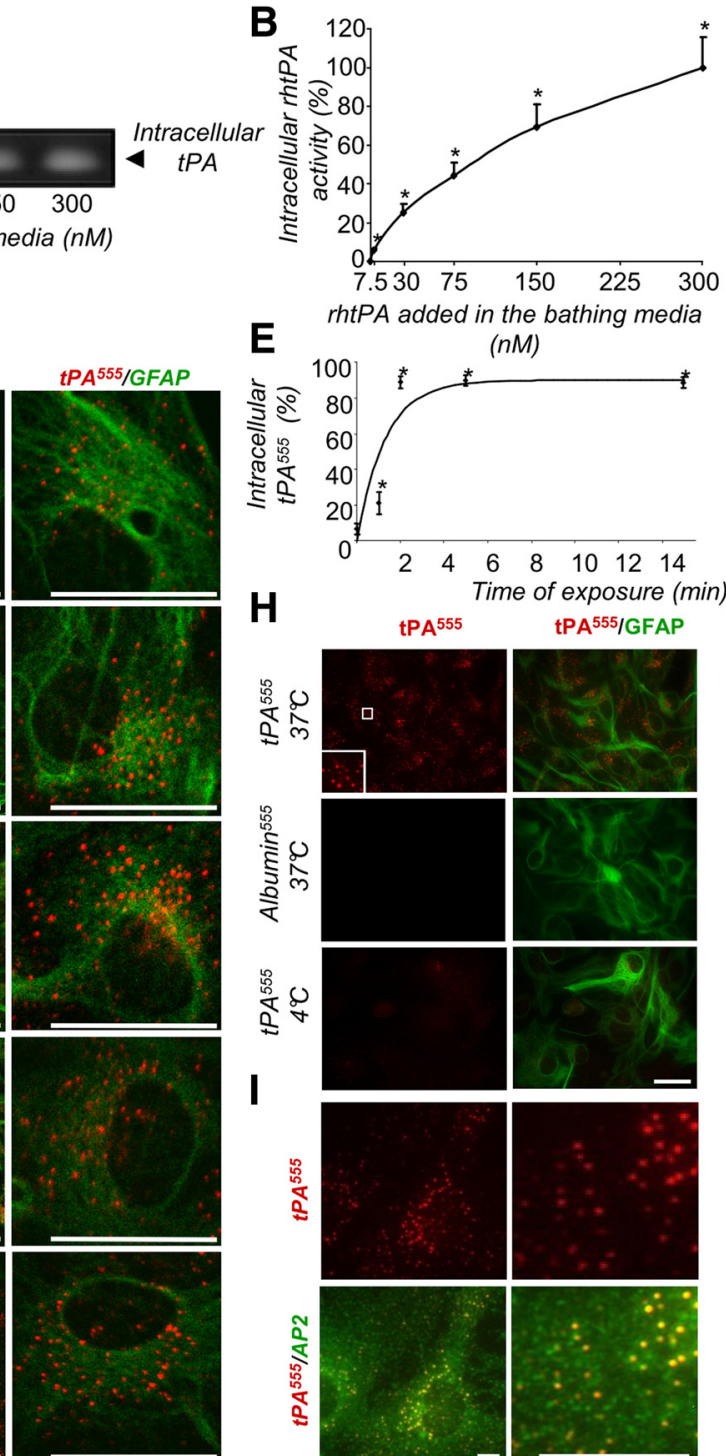

E
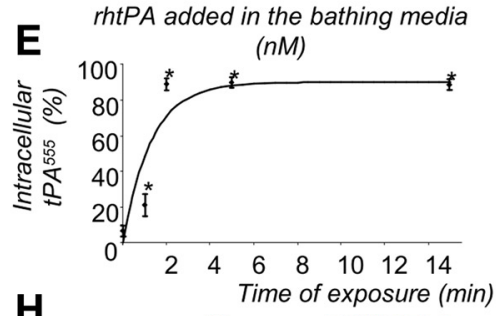

H

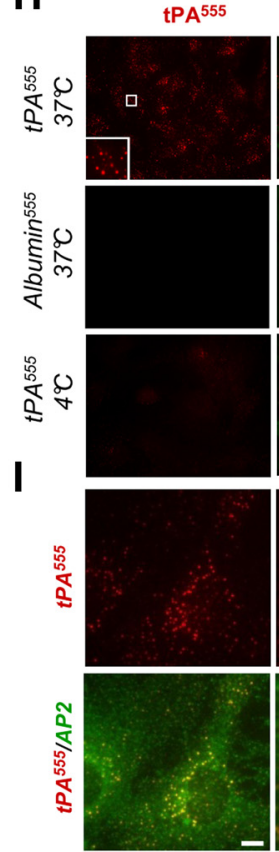

tPA $^{555 / G F A P ~}$
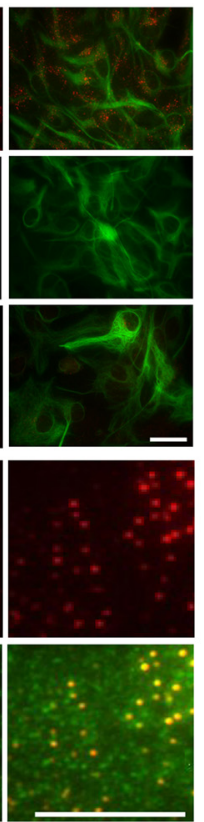

C

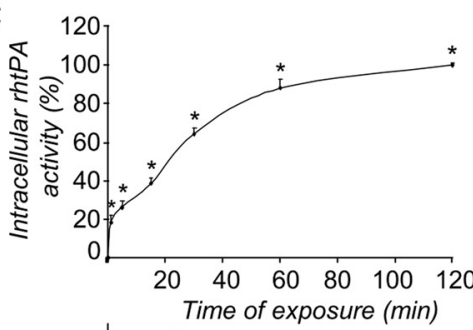

$\mathbf{F}$

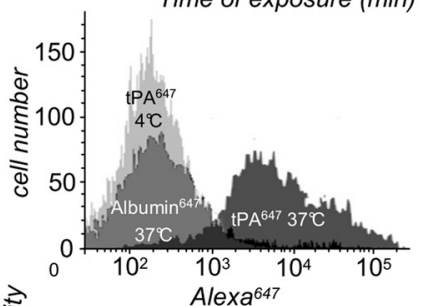

J

G
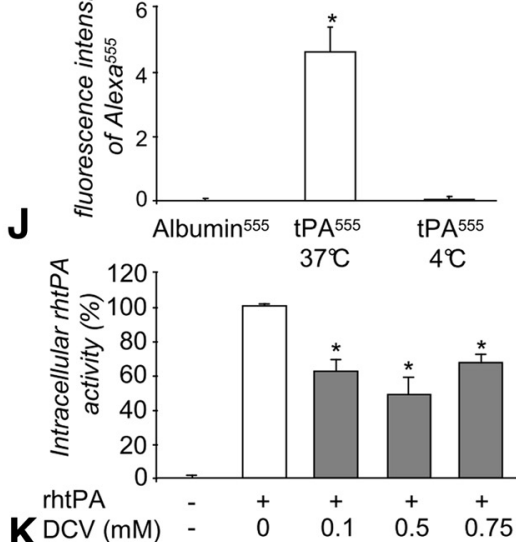

$\begin{array}{llllll}\mathbf{K} D C V(\mathrm{mM}) & - & 0 & 0.1 & 0.5 & 0.75\end{array}$

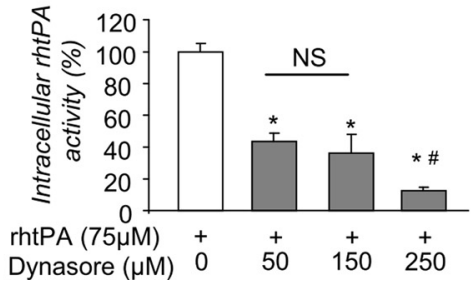

Figure 1. tPA is constitutively endocytosed by astrocytes via a clathrin-dependent and dynamin-dependent process. $A$, Zymography assay (representative image out of triplicate) for proteolytic activity of intracellular tPA in protein extracts from cell monolayer of astrocytes exposed to exogenously added rhtPA (from 7.5-300 nM, as indicated) for 15 min. B, Graph shows mean \pm SEM $(n=4)$ of quantification (enzymatic assay) of intracellular tPA proteolytic activity calculated as percentage of intracellular tPA activity at $300 \mathrm{~nm}$ exogenous rhtPA in protein extracts from cell monolayer of astrocytes treated as in $A$. ${ }^{*}$, Significantly $(p<0.05)$ different from control. C, Astrocyte cultures were exposed to rhtPA (75 nm) for $15-120$ min. Graph shows mean \pm SEM $(n=3)$ of quantification (enzymatic assay) of intracellular tPA proteolytic activity calculated as percentage of activity at 120 min. ${ }^{*}$, Significantly $(p<0.05)$ different from control. $\boldsymbol{D}$, Astrocyte cultures were exposed to $\mathrm{PAA}^{555}(75 \mathrm{nM})$ for $15-120 \mathrm{~min}$, as indicated. Photomicrographs (representative image of $n=3$ ) show tPA ${ }^{555}$ staining (left, red) and merged image with GFAP immunoreactivity (middle, red and green). Right, Magnification of selected regions defined by inserts. $\boldsymbol{E}$, Graph shows mean \pm SEM ( $n=3$ ) quantification of sctPA ${ }^{555}$ fluorescence in SDS-PAGE cell monolayer of astrocytes normalized to bound plus internalized tPA ${ }^{555}$ in single-cycle endocytosis experiment (see Materials and Methods). ${ }^{*}$, Significantly $(p<0.05)$ different from beginning of the experiment. $E$, Astrocyte cultures were exposed for 15 min to tPA ${ }^{555}$, at $4^{\circ} \mathrm{Cor} 37^{\circ} \mathrm{C}$, or to albumin ${ }^{555}(75 \mathrm{~nm})$ at $37^{\circ} \mathrm{C}$, as indicated. $\boldsymbol{F}$, Graph shows mean \pm SEM $(n=3)$ of the quantification of the fluorescence of Alexa ${ }^{555}$ per $100 \mu \mathrm{m}^{2} .{ }^{*}$, Significantly $(p<0.05)$ different from control. $\mathbf{G}$, Astrocytes were exposed for 15 min to $\mathrm{PAA}^{647}$, at $4^{\circ} \mathrm{C}$ or $37^{\circ} \mathrm{C}$, or to albumin ${ }^{647}$ at $37^{\circ} \mathrm{C}$. Graph (representative image of $n=3$ ) shows the flow cytometry analysis of fluorescence intensity of Alexa ${ }^{647}$ in astrocytes. $\boldsymbol{H}$, Astrocyte cultures were exposed for $15 \mathrm{~min}$ to $\mathrm{tPA}^{555}$. Photomicrographs show (representative image of triplicate) tPA ${ }^{555}$ staining (top, red) and immunocolocalization (bottom) with AP-2 (green). I, Astrocyte cultures were exposed to rhtPA (75 nM) with or without DCV (from 0.1-0.75 mM, as indicated) for 15 min. Graph shows mean \pm SEM $(n=3$ ) of quantification (enzymatic assay) of intracellular tPA proteolytic activity calculated as percentage of activity in the absence of DCV. ${ }^{*}$, Significantly $(p<0.05)$ different from control. $\boldsymbol{J}$, Astrocyte cultures were exposed to rhtPA (75 nM) for $15 \mathrm{~min}$ after preincubation with or without dynasore (from $50-250 \mu \mathrm{M}$, as indicated) for 30 min. $K$, Graph shows mean \pm SEM $(n=3)$ of quantification (enzymatic assay) of intracellular tPA proteolytic activity calculated as percentage of activity in the absence of dynasore. ${ }^{*}$,, Significantly $(p<0.05)$ different from control or dynasore conditions (50 and $150 \mu \mathrm{M}$ ). ns, not significant. Scale bars, $30 \mu \mathrm{m}$.

clathrin-dependent, and dynamin-dependent endocytosis; (2) astrocytes are able to release tPA; (3) extracellular glutamate inhibits the release of tPA by astrocytes; and (4) this inhibitory action of glutamate is mediated by kainate receptor-induced protein kinase C (PKC) signaling. Finally, this study demonstrates the role played by astrocytes in regulating the levels of extracellular tPA.

\section{Materials and Methods}

\section{Materials}

Recombinant human tPA (rhtPA; Actilyse) was purchased from Boehringer Ingelheim. Receptor associated-protein (RAP) was provided from Gentaur. DMEM, poly-D-lysine, laminin, glutamine, cytosine $\beta$-D-arabinoside, albumin, L-glutamic acid monosodium, AMPA, kainic acid monohydrate, 6-cyano-7nitroquinoxaline-2,3-dione (CNQX), mono-dansyl-cadaverine (DCV), dyna- 
sore, human $\alpha$-2-macroglobulin, and PBS were purchased from Sigma-Aldrich. NMDA, MK-801, bisindolymaleimide I (bis), 2,3-dihydroxy-6-nitro-7sulfonyl-benzo[f] quinoxaline (NBQX), (RS)-1-amino-5-phosphonoindan-1-carboxylic acid (APICA), (RS)-1-aminoindan-1,5-dicarboxylic acid (AIDA), (RS)- $\alpha$-cyclopropyl-4-phosphonophenylglycine (CPPG), and $(\alpha S)$ - $\alpha$-Amino-3-[(4-carboxyphenyl)methyl]-3,4-dihydro-5-iodo-2,4-dioxo1(2H)-pyrimidinepropanoic acid (UBP 301) were purchased from Tocris Bioscience. Alexa fluor 555 and 647 carboxylic acid, succinimidyl ester, LysoTracker, fetal bovine serum (FBS), and horse serum (HS) were purchased from Invitrogen.

\section{Animals and surgery}

Mice were housed in a temperature-controlled room on a $12 \mathrm{~h}$ light/dark cycle with food and water ad libitum. Experiments were performed in accordance with French ethical laws (Act No. 87-848, Ministère de l'Agriculture et de la Forêt) and European Communities Council Directives of November 24, 1986 (86/609/EEC), guidelines for the care and use of laboratory animals.

\section{Plasmid constructions and production of recombinant proteins}

Plasmids. pEGFP-tetanus neurotoxin-insensitive vesicle-associated membrane protein (TI-VAMP), pEGFP-TI-VAMP ${ }_{\text {DN }}$, and pEGFPVAMP-3 (Martinez-Arca et al., 2000); pCMV5-TeNT ${ }^{-}$and pCMV5$\mathrm{TeNT}^{+}$(McMahon et al., 1993); pEGFP-Rab5 and pRab5 ${ }_{\mathrm{DN}}$ (kind gift from Letizia Lanzetti, University of Turin, Turin); pEGFP-CD63 (kind gift from John Paul Luzio, Cambridge University, Cambridge); and pEGFP-VAMP-4 were previously described (Mallard et al., 2002).

pSynapsin fluorescent tPA. Coding region of tPA, including its signal peptide, was cloned in $\mathrm{pET}-161$ plasmid vector (Invitrogen) to introduce a fluorescent tag, lumio, at the C-terminal end. Fluorescent tPA (tPAlumio) was then transferred to pSynapsin (pSyn) plasmid vector (a kind gift from Dr. Streamson C. Chua, Columbia University, NY) to form pSyn-tPA-lumio.

Production of recombinant mutants of $t P A$ and of inactive $t P A$. The wt-tPA and $\triangle$ EGF-tPA recombinant proteins were obtained as previously described from the Rattus norvegicus tPA cDNA (Swiss-Prot accession P19637). The $\Delta \mathrm{F}-\mathrm{tPA}$, with a deletion of the finger domain, was generated following the same method, by using the upstream primer $5^{\prime}$ CCGGGATCCCCCGTCCGAAGTTGC- $3^{\prime}$ and the downstream primer 5'-GGCAAGCTTTTGCTTCATGTTGTCTTGAATCCAGTT-3', thus amplifying a sequence corresponding to tPA lacking the finger domain. For obtaining inactive tPA, purified rhtPA was mixed with the irreversible inhibitor glu-gly-arg-chloromethyl ketone (GGACK, Calbiochem) for $4 \mathrm{~h}$ at $4^{\circ} \mathrm{C}$ with continuous stirring. The resulting solution was dialyzed (Slide-A-Lyser $10 \mathrm{KDa}$, Thermo Fisher Scientific) in bicarbonate buffer $\left(0.1 \mathrm{M} \mathrm{NaHCO}_{3}\right.$ and Tween $80(0.1 \%), \mathrm{pH} 8.4$,overnight at $4^{\circ} \mathrm{C}$ to remove free GGACK. The inactive tPA was labeled with Alexa ${ }^{555}$ as described below.

\section{Labeling of tPA, albumin and $\alpha$-2-macroglobulin with Alexa ${ }^{555}$ and Alexa 647}

Arginine content in Actilyse was removed by dialysis at $4^{\circ} \mathrm{C}$ overnight (see above). Then, purified rhtPA was mixed with the $N$-succinimidyl ester of Alexa ${ }^{555}$ or Alexa ${ }^{647}$ for $4 \mathrm{~h}$ at $4^{\circ} \mathrm{C}$ with continuous stirring. The resulting solution was dialyzed in bicarbonate buffer overnight at $4^{\circ} \mathrm{C}$ to remove unbound dyes. Afterward, tPA-Alexa ${ }^{555}$ ( $\mathrm{tPA}^{555}$ ) or tPAAlexa ${ }^{647}\left(\mathrm{tPA}^{647}\right)$ were frozen and stored at $-80^{\circ} \mathrm{C}$ until further use. The same procedure was performed with albumin and $\alpha$-2-macroglobulin.

\section{Antibodies}

Primary antibodies used were as follows: for immunocytochemistry, mouse polyclonal anti-GFAP, mouse monoclonal anti-adaptor protein 2 (anti-AP-2) antibodies (1:1000 and 1:500 dilution, respectively; Abcam), and rabbit monoclonal anti-LRP1 antibodies directed against the $\mathrm{C}$ terminus of LRP1 (1:500; a kind gift from Professor Dudley K. Strickland, University of Maryland School of Medicine, Baltimore, MD); for immunoblots, goat polyclonal anti-tPA antibodies (1:1000, Santa Cruz Biotechnology). Secondary antibodies were as follows: for immunocytochemistry, $\mathrm{F}\left(\mathrm{ab}^{\prime}\right) 2$ fragments of donkey anti-mouse IgG linked to FITC or Alexa ${ }^{649}$, and $\mathrm{F}\left(\mathrm{ab}^{\prime}\right) 2$ fragments of donkey anti-rabbit linked to FITC (1:300, Jackson

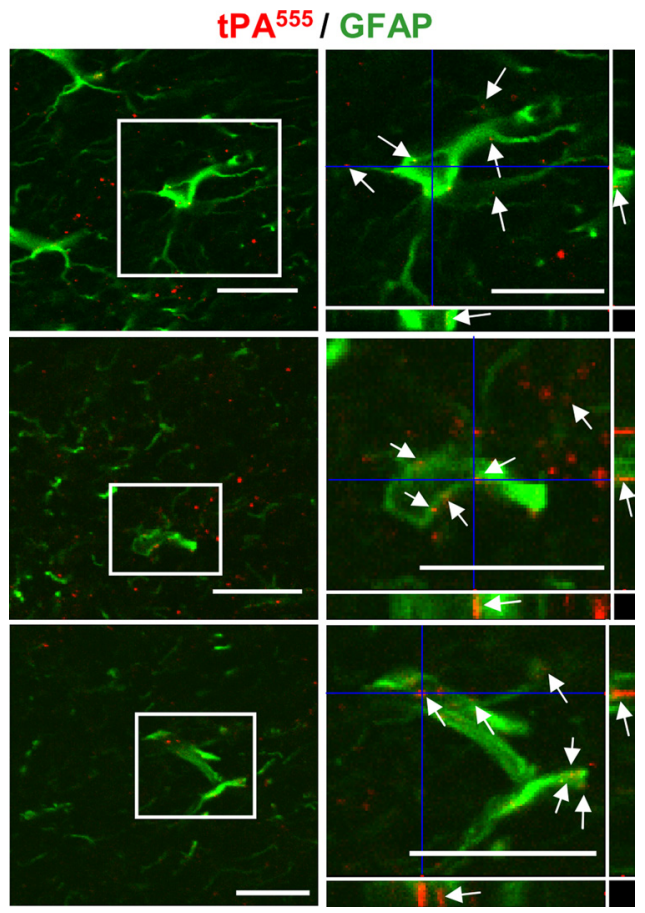

Figure 2. Astrocytes uptake TPA in vivo. Mice were injected stereotaxically into the cortex with $1 \mu \mathrm{g}$ of tPA ${ }^{555}$. Confocal photomicrographs show three representative merged images of brain sections with $\mathrm{TPA}^{555}$ staining and GFAP immunoreactivity (left, red and green). Right, Magnification of selected regions defined by insert. Photomicrographs correspond to a transversal plan $(0.5 \mu \mathrm{m})$ of the all stack images $(10 \mu \mathrm{m})$ acquired with a confocal microscope. Scale bars, $30 \mu \mathrm{m}$. Arrows indicate staining for $\mathrm{PPA}^{555}$ in GFAP ${ }^{+}$astrocytes.

ImmunoResearch); for Western blots, anti-goat peroxidase-conjugated (Sigma-Aldrich).

Primary neuronal cultures, primary astrocyte cultures and mixed cultures of neurons and astrocytes

Primary neuronal cultures, primary astrocyte cultures and mixed cultures of neurons and astrocytes were obtained as previously described (Nicole et al., 2001). For immunocytochemistry, cells were cultured on glass-bottom Petri dishes (MatTek) coated with poly-D-lysine $(0.1 \mathrm{mg} /$ $\mathrm{ml})$ and laminin $(0.02 \mathrm{mg} / \mathrm{ml})$. Neuronal cultures after $14 \mathrm{~d}$ in vitro (DIV) were used for toxicity experiments and at 12 DIV for neuronal tPA-lumio recording experiments.

\section{Neuronal toxicity assay}

Slowly triggered excitotoxicity was induced at $37^{\circ} \mathrm{C}$ by a $24 \mathrm{~h}$ exposure to NMDA $(12.5 \mu \mathrm{M})$ in serum-free DMEM supplemented with glycine (10 $\mu \mathrm{M}, \mathrm{MS}$-glycine). Neuronal death was assessed by phase-contrast microscopy and quantified by measurement of the activity of lactate dehydrogenase (LDH) released by damaged cells into the bathing medium with a cytotoxicity detection kit (Roche Diagnostics). The maximum neuronal death was determined in sister wells exposed to NMDA (500 $\mu \mathrm{M})$ for $24 \mathrm{~h}$ in MS-glycine. Background LDH levels were determined in sister wells subjected to control washes and subtracted from experimental values to yield the signal specific to experimentally induced injury.

\section{Transfections}

Plasmids were transfected in cultures of astrocytes by using Lipofectamine 2000 ( $2 \mu \mathrm{g}$, Invitrogen), in HEPES and bicarbonate buffered saline solution (HBBSS) containing the following (in $\mathrm{mM}$ ): $116 \mathrm{NaCl}, 5.4$ $\mathrm{KCl}, 1.8 \mathrm{CaCl}$, $0.8 \mathrm{MgSO}_{4} 1.3 \mathrm{NaH}_{2} \mathrm{PO}_{4}, 12$ HEPES, 5.5 glucose, 25 bicarbonate, and $10 \mu \mathrm{M}$ glycine, $\mathrm{pH} 7.45$, at $37^{\circ} \mathrm{C}$. After $36 \mathrm{~h}$, cultures were washed in HBBSS at $37^{\circ} \mathrm{C}$ and treated with tPA-Alexa ${ }^{555}$ for different amounts of times. Then, cells were washed in PBS $(0.1 \mathrm{M})$ and fixed in paraformaldehyde (PFA, 4\%) dissolved in PBS $(0.1 \mathrm{~m})$ for $30 \mathrm{~min}$ at $4^{\circ} \mathrm{C}$. 


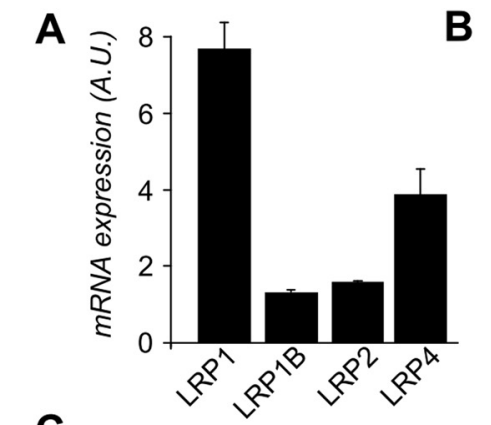

$$
\text { C }
$$

tPA555
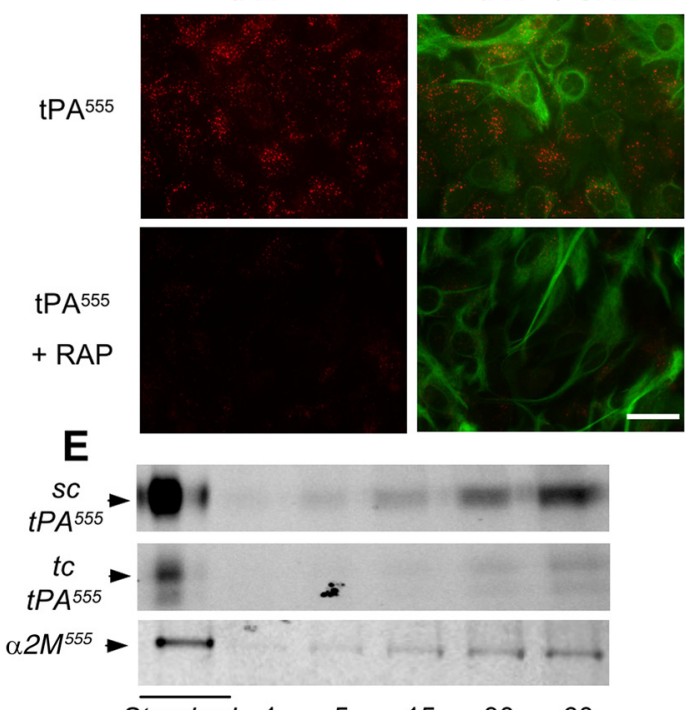

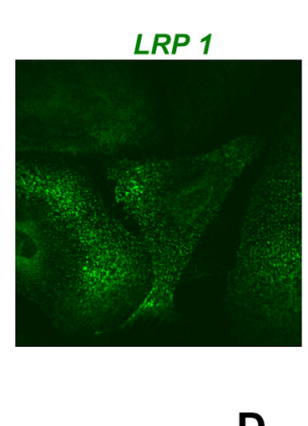

D
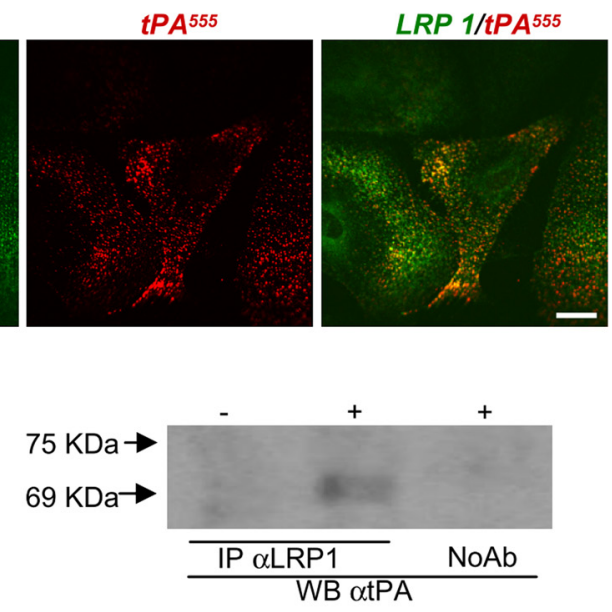

G

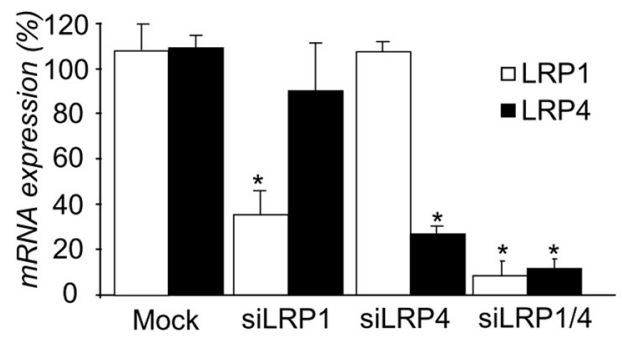

H

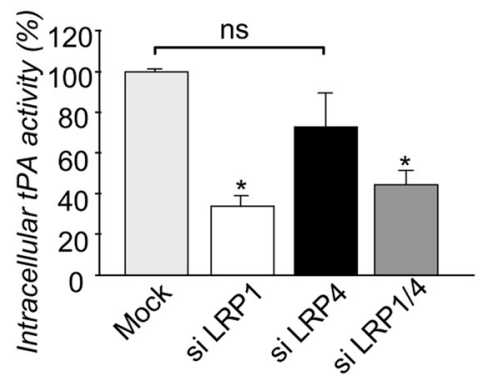

$20 \quad$ Time of exposure $(\mathrm{min})$

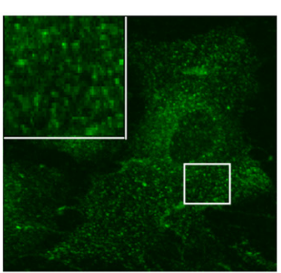

LRP 1

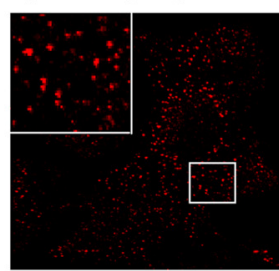

tPA 555

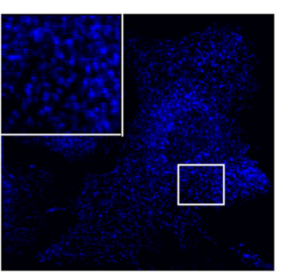

AP2

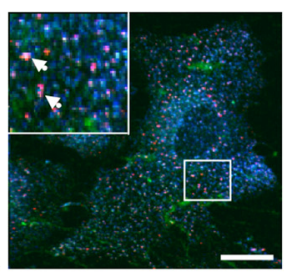

LRP 1/tPA555/AP2

Figure 3. Endocytosis of tPA by astrocytes is mediated by LRP1. $A$, Quantitative PCR for relative levels of LRP $m R N A$ (mean $\pm S E M, n=3$ ) in astrocyte cultures, computed by calculating the $2^{-\Delta \Delta \mathrm{ct}}$. The housekeeping gene expression (cyclophilin), used for normalization, was not influenced by the stages in vitro. B, Astrocyte cultures were exposed for 15 min to tPA ${ }^{555}$ (75 $\mathrm{nm)}$. Photomicrographs (representative image of $n=4$ ) show staining for immunocolocalization of LRP1 (green) and tPA ${ }^{555}$ (red) viewed individually and as a merged image, as indicated (right, green and red). C, Astrocyte cultures were exposed for $15 \mathrm{~min}$ to tPA ${ }^{555}(75 \mathrm{~nm})$ alone or with RAP $(0.5 \mu \mathrm{M})$. Photomicrographs (representative image of $\left.n=4\right)$ show tPA ${ }^{555}$ (left, red) viewed individually, or as a merged image with immunostaining for astrocytes marker, GFAP (right, red and green). D. Cultured astrocytes were exposed or not to tPA (75 nM) for 15 min and their intracellular protein extract was allowed to react with anti-LRP1 antiserum. The LRP1-immunoprecipitated proteins were analyzed with anti-tPA antiserum (WB $\alpha$ tPA) (representative image of $n=3)$. A control with no anti-LRP1 antiserum addition was included (NoAb). E, Astrocyte cultures were exposed to $\mathrm{tPA}^{555}$ (7.5 nM) or $\alpha$-2-macroglobulin ${ }^{555}\left(\alpha 2 \mathrm{M}^{555}, 7.5 \mathrm{~nm}\right)$ for 1-60 min. SDS-PAGE (representative of $n=5)$ analysis of intracellular protein extracts shows $\mathrm{TPA}^{555}$ and $\alpha$-2-macroglobulin ${ }^{555}$ in cell monolayer of astrocytes. sc-tPA and tc-tPA were detectable. $\boldsymbol{F}$, Graph shows mean \pm SEM ( $n=$ 5) quantification of total tPA ${ }^{555}$ (sc-tPA plus tc-tPA, solid line) and $\alpha$-2-macroglobulin ${ }^{555}$ (dotted line) fluorescence in SDS-PAGE cell monolayer of astrocytes treated in $\boldsymbol{E}$. ${ }^{*}, \#$, Significantly $(p<$ 0.05 ) different from the beginning of the experiment, for tPA ${ }^{555}$ and $\alpha 2 \mathrm{M}^{555}$, respectively. G. Astrocytes were transfected with negative control (mock) siRNA against LRP1, LRP4, or LRP1 and LRP4. Graph shows mean \pm SEM $(n=3)$ of relative expression of mRNA levels of LRP1 and LRP4. * , Significantly different $(p<0.05)$ from mock condition. $\boldsymbol{H}$, Astrocyte cultures transfected with mock siRNA against LRP1, LRP4, or LRP1 and LRP4 were exposed for 15 min to rhtPA (75 nM). Graph shows mean \pm SEM $(n=4)$ of quantification (enzymatic assay) of proteolytic activity of intracellular tPA calculated as percentage of mock condition. *, Significantly different $(p<0.05)$ from mock condition. ns: not significant. $I$, Astrocyte cultures were exposed for 15 min to tPA ${ }^{555}$ (75 nM). Photomicrographs (representative image of $n=3$ ) show LRP1 immunoreactivity (green), $\mathrm{PAA}^{555}$ staining (middle, red), AP-2 immunoreactivity (middle, blue), and merged image (right, green, red and blue). Arrows show immunocolocalization of LRP1, AP-2, and tPA ${ }^{555}$ (inserts show magnification of the selected regions). Scale bars, $20 \mu \mathrm{m}$. 
After final rinses in PBS $(0.1 \mathrm{M})$, cells were visualized in an Eclipse (TE2000-E) inverted C1 confocal microscope (Nikon).

\section{Immunocytochemistry}

After treatment, astrocyte cultures were washed with PBS, fixed in PFA for $30 \mathrm{~min}$ at $4^{\circ} \mathrm{C}$, washed in PBS $(0.1 \mathrm{M})$, and blocked $1 \mathrm{~h}$ in PFA (4\%) dissolved in PBS (0.1 M). Immunocytochemistry was performed by standard procedure. For standard epifluorescence microscopy, images were digitally captured using a DM6000 microscope-coupled CoolSNAP camera (Leica) and visualized with Metavue software (Molecular Devices). Confocal laserscanning microscopy was assessed using a Nikon TE2000-E confocal microscope.

\section{Single-cycle endocytosis experiments}

Astrocyte cultures were serum starved for $1 \mathrm{~h}$ in ice-cold DMEM and then exposed to tPA ${ }^{555}$ (100 nM) for $15 \mathrm{~min}$ at $4^{\circ} \mathrm{C}$. Then, astrocytes were washed twice in cold PBS $(0.1 \mathrm{M})$ and cultures were incubated at $37^{\circ} \mathrm{C}$ in fresh DMEM for various times $(0-15 \mathrm{~min})$. Cells were then washed with ice-cold acid buffer $(0.2 \mathrm{M}$ acetic acid, $0.5 \mathrm{M} \mathrm{NaCl}$, and $10 \mu \mathrm{M}$ rhtPA, pH 2.5) and medium was collected to determine the amount of surface-bound tPA ${ }^{555}$. Finally, cultures were washed twice with cold PBS and monolayer protein extracts were collected to determine the amount of internalized tPA. Bathing media and protein extracts from cell monolayers were subjected to SDS-PAGE (10\%). $\mathrm{tPA}^{555}$ was visualized using an ImageQuant LAS 4000 camera. Results are presented as the ratio of internalized tPA and the sum of internalized tPA plus surface-bound tPA (surface-bound tPA corresponds to total cell-bound tPA).

\section{Fluorescence-activated cell sorting}

Cells were washed with PBS $(0.1 \mathrm{M})$ and detached with trypsin $(0.5 \%)$. The cell suspension was washed with DMEM containing FBS (10\%), HS $(10 \%)$, and glutamine $(1 \%)$ to neutralize trypsin, and then with PBS. Cells were fixed in PFA for $10 \mathrm{~min}$ on ice and washed with PBS $(0.1 \mathrm{M})$. Using fluorescence-activated cell sorting (FACS), cellular fluorescence was measured immediately after fixation with a FACSCanto II flow ctyometer (Becton Dickinson). Alexa ${ }^{647}$ was identified by using a $660 \mathrm{~nm}$ bandpass filter and the background level was estimated with untreated cells. A primary gate based on physical parameters (forward and side light scatter) was set to exclude dead cells or cell debris. Analyses were performed in FACS Diva Software (Becton Dickinson).

\section{Protein extractions}

Cells were lysed at $4^{\circ} \mathrm{C}$ in Tris- $\mathrm{HCl} 50 \mathrm{~mm}, \mathrm{pH} 7.4, \mathrm{NaCl} 150 \mathrm{~mm}$, and $0.5 \%$ Triton X-100 (TNT buffer) for $1 \mathrm{~h}$. To clear lysates, samples were centrifuged for $15 \mathrm{~min}(12,000 \mathrm{~g})$ at $4^{\circ} \mathrm{C}$. Proteins were quantified by using BCA protein assay (Pierce).

\section{tPA activity measurement}

Enzymatic assay. Fluorogenic substrate (5 $\mu \mathrm{M}$, Spectrozyme XF444, American Diagnostica) was incubated with proteins $(15 \mu \mathrm{g})$. Measurements were performed at $37^{\circ} \mathrm{C}$ using a multiplate reader (Chameleon, Hidex).

Casein-plasminogen zymography assay. Zymography was performed as previously described (Fernandez et al., 2004).

\section{Immunoblotting}

Immunoblotting was performed following a standard procedure. After incubation with the secondary antibodies, proteins were visualized with an enhanced chemiluminescence using ImageQuant LAS 4000 camera (GE Healthcare).
B

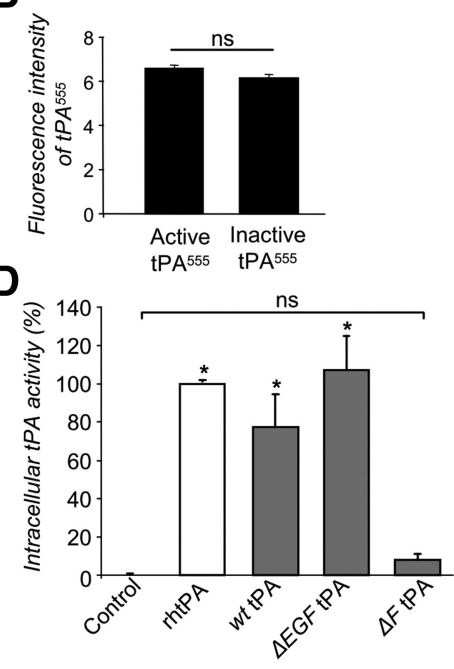

Figure 4. TPA finger domain is critical for astrocytic endocytosis. $\boldsymbol{A}$, Schematic representation of the recombinant wt $t P A$, 列 .ns: not significant. C, Zymography analysis (representative of triplicate) of intracellular tPA proteolytic activity of proteins SEM $(n=3)$ of quantification (enzymatic assay) of intracellular tPA proteolytic activity (percentage of rhtPA condition). rent from rhtPA condition. ns: not significant.

\section{Immunoprecipitation}

Proteins were immunoprecipitated at $4^{\circ} \mathrm{C}$ overnight using anti-LRP1. The immunoprecipitated complexes were incubated for $1 \mathrm{~h}$ at $4^{\circ} \mathrm{C}$ with Dynabead ProteinG (Invitrogen). After collection, the beads were collected on magnetic separator and washed three times with TNT. The immunoprecipitated proteins were analyzed by immunoblotting.

\section{Extraction of total RNA}

Total RNAs were extracted from cultured cells by using the NucleoSpin RNA II kit (Macherey-Nagel) according to the manufacturer's instructions.

\section{Quantitative real-time PCR}

Total RNAs $(1 \mu \mathrm{g})$ from each sample were reverse-transcribed using the iScript Select cDNA Synthesis Kit (Bio-Rad). Primers were designed for each gene using the Beacon Designer software (Bio-Rad). Primer alignments were performed with the BLAST database to ensure the specificity of primers. PCR reagents were prepared with RNase-free water-containing primers and IQ SYBR Green Supermix (Biorad). For PCR amplification, mix $(20 \mu \mathrm{l})$ was added to reverse transcription reaction $(5 \mu \mathrm{l})$ previously diluted (1:20). Two negative controls were performed during each quantitative PCR (qPCR) experiment: reactions without reverse transcription to confirm absence of genomic DNA contamination, and samples with no added cDNA template to prove the absence of primer dimers. Assays were run in triplicate on the Chromo 4 Real-Time PCR detector (Bio-Rad). Amplification conditions were as follows: Hot Goldstar enzyme activation, $95^{\circ} \mathrm{C}$ for $3 \mathrm{~min} ; 50$ cycles of PCR (denaturation: $95^{\circ} \mathrm{C}, 15 \mathrm{~s}$, and hybridation/extension $60^{\circ} \mathrm{C}, 1 \mathrm{~min}$ ). Cyclophilin A was used as a housekeeping gene. The levels of expression of gene of interest were computed as follows: relative mRNA

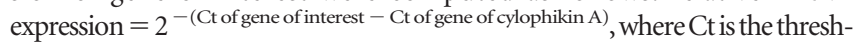
old cycle value.

\section{Sequence and protocol of silencing $m R N A$}

Six stealth siRNA duplex oligoribonucleotides against LRP1 and LRP4 receptors were tested (Invitrogen). The sequences were as follows: LRP1_01 sense UGGCUGACGGGAAACUUCUACUUUG LRP1_01 antisense CAAAGUAGAAGUUUCCCGUCAGCCA, LRP1_02 sense CACACCCAUUUGCCGUGACACUGUA LRP1_021 antisense UACAGUGUCACGGCAAAUGGGUGUG, LRP1_03 sense CCAAGGU GUGAGGUGAACAAGUGUA LRP1_03 antisense UACACUUGUUCA CCUCACACCUUGG,LRP4_01 senseACGGGUGAGGAGAACUGCAA UGUUA LRP4_01 antisense UAACAUUGCAGUUCUCCUCACCCGU, 

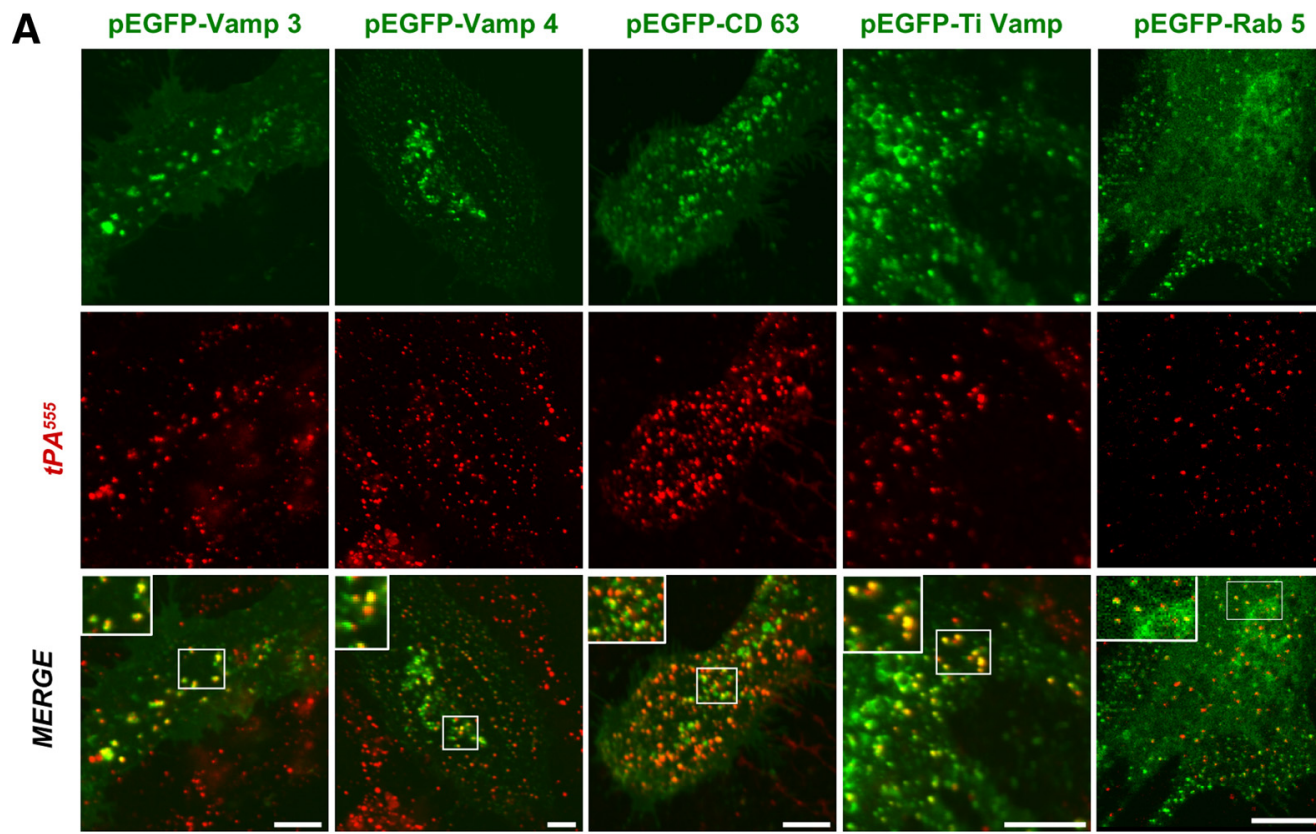

B

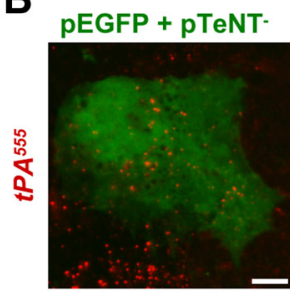

pEGFP + pTeNT+
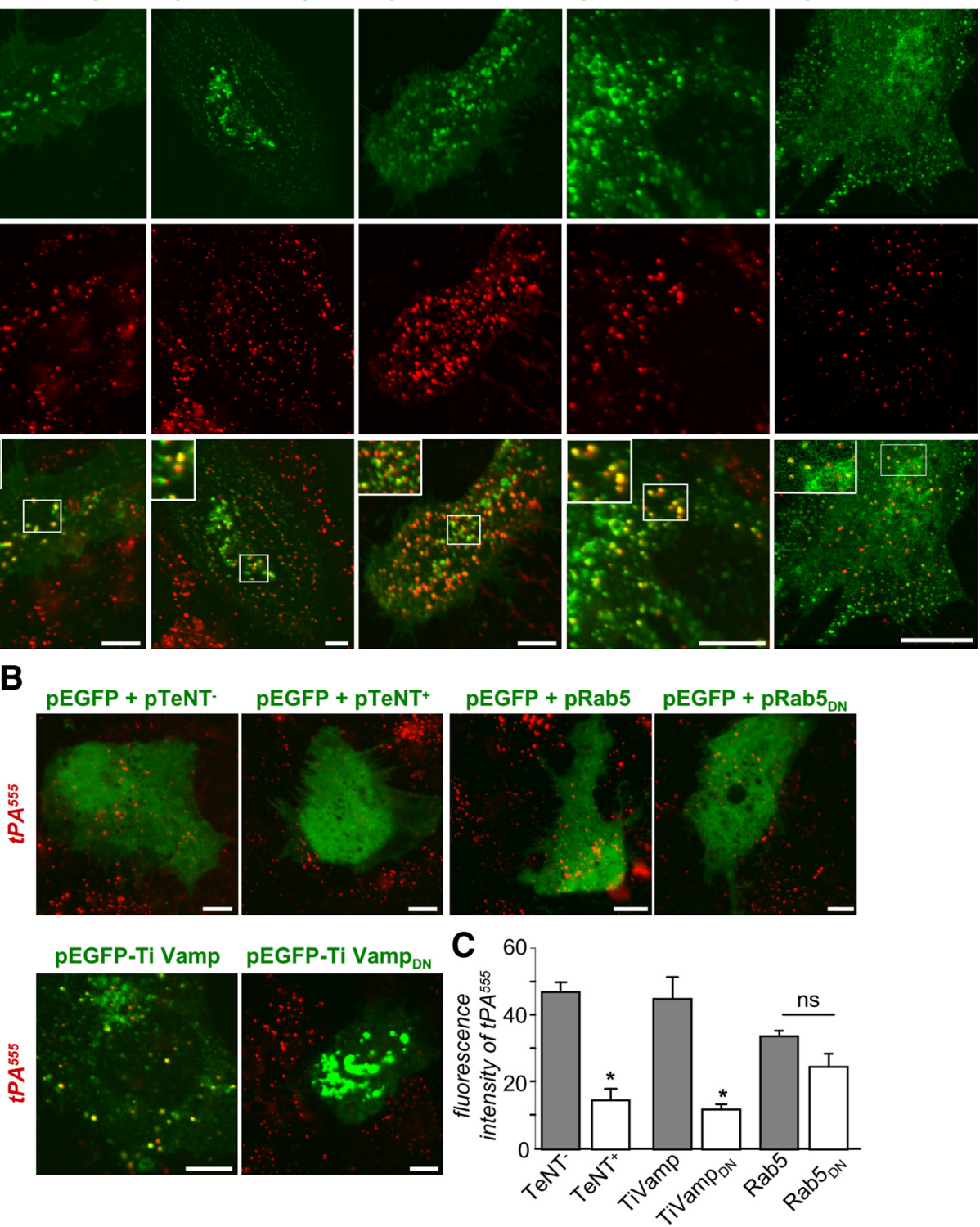

Figure 5. Intracellular trafficking of PAA in astrocytes. A, Astrocyte cultures were transfected with pEGFP-Rab5, pEGFP-VAMP3, pEGFP-CD63, pEGFP-TIVAMP, or pEGFP-VAMP-4 vectors. After 36h of transfection, astrocytes were treated for 15 min with $\mathrm{APA}^{555}$ (75 nM). Photomicrographs (representative image of triplicate) show GFP staining (top, green), tPA ${ }^{555}$ (middle, red), and merged image (bottom, green and red). Inserts show magnification of the selected regions. B, Astrocyte cultures were transfected with pEGFP with pCMV5-TeNT ${ }^{+}$vector, pEGFP with pCMV5-TeNT - vector,

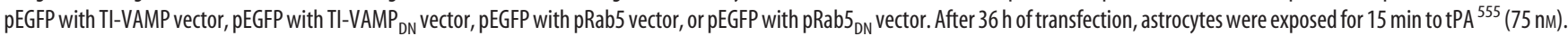
Photomicrographs (representative image of $n=3$ ) show merged image of GFP (green) and IPA (red). Scale bars, $30 \mu \mathrm{m}$. C, Graph shows mean \pm SEM $(n=3)$ of quantification of fluorescence intensity of $\mathrm{tPA}^{555}$ per $100 \mu \mathrm{m}^{2}$. ${ }^{*}$, Significantly $(p<0.05)$ different from control.

LRP4_02 sense CCACAGGUAUCAACCUGCUACGAGA LRP4_02 antisense UCUCGUAGCAGGUUGAUACCUGUGG,LRP4_03 senseCAGCCGUGUA UAAUCAGCUGUGCUA LRP4_03 antisense UAGCACAGCUGAUUAUAC ACGGCUG.

Stealth siRNA oligoribonucleotides ( $40 \mathrm{~nm}$ ) were transfected into primary cultures of astrocytes by using Lipofectamine 2000 ( $2 \mu \mathrm{g}$, Invitrogen). The Stealth RNA negative Control Duplex (Invitrogen) was used as a mock condition. Cultures were replaced with MS-glycine after the transfection and astrocytes were treated $48 \mathrm{~h}$ later. After characterization by qPCR, we selected from among the six stealth siRNA oligo duplexes against LRP1 and LRP4 receptors, the one that showed the most significant knockdown effect ( $>60 \%$ of reduction of mRNA expression).

\section{Neuronal tPA lumio recording}

Plasmid pSyn-tPA-lumio was transfected in mixed cultures of neurons and astrocytes (12 DIV) by using Lipofectamine 2000 ( $2 \mu \mathrm{g}$, Invitrogen), in $\mathrm{HBBSS}$ at $37^{\circ} \mathrm{C}$. After $36 \mathrm{~h}$, mixed cultures were washed in HBBSS at $37^{\circ} \mathrm{C}$ and incubated with lumio green reagent (Lumio Green In-Cell Detection Kit, Invitrogen) for 5 min. Then, cells were washed in HBBSS and incubated with Disperse Blue 3, according to the manufacturer's protocol. Observations were performed with the Nikon confocal microscope. Time-lapse acquisitions were performed each minute for $3 \mathrm{~h}$. At $\mathrm{t}=0$ (30 min after the beginning of the acquisition), bicuculline $(50 \mu \mathrm{M})$ and 4-amino-pyrimidine (4-AP, $2.5 \mathrm{~mm}$ ) or vehicle were introduced in the Petri dish. In some experiments, CNQX $(10 \mu \mathrm{M})$ was added at $\mathrm{t}=60$ min. Quantification of fluorescence was performed using the Nikon EZ-C1 software.

\section{tPA follow-up assay}

First, astrocytes cultures were exposed for $1 \mathrm{~h}$ to $\mathrm{tPA}^{555}$ (300 nM), or albumin ${ }^{555}$ ("loading"). Then, cells were washed three times in PBS (0.1 M) at $37^{\circ} \mathrm{C}$ and medium was replaced by MS-glycine ("release"). Finally, bathing media and protein extracts from cell monolayers were collected 
A

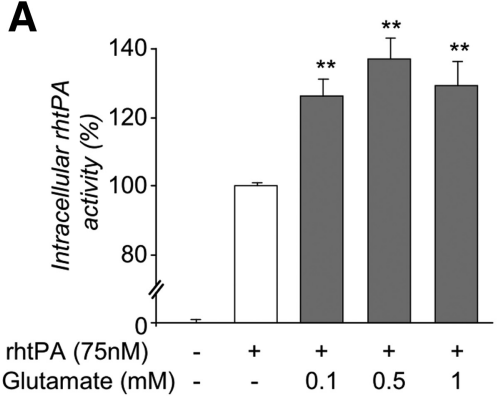

B

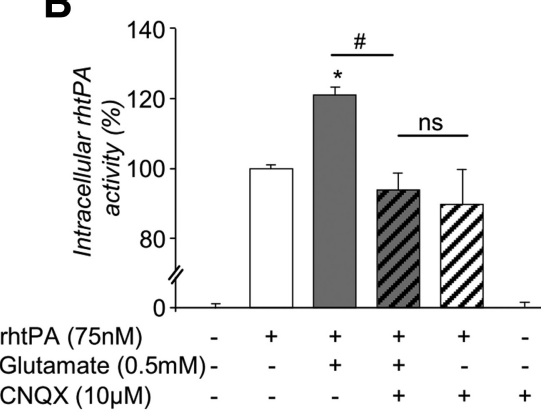

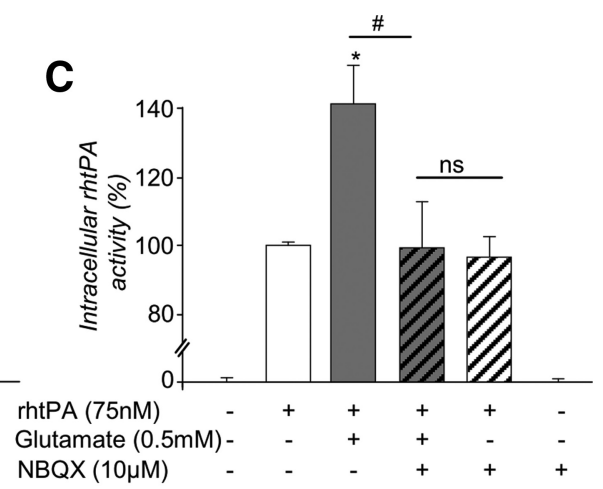

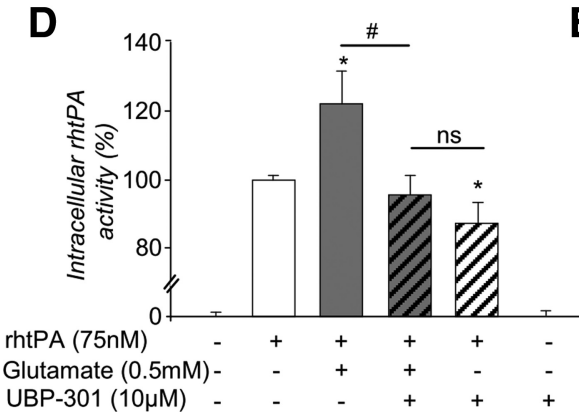

E
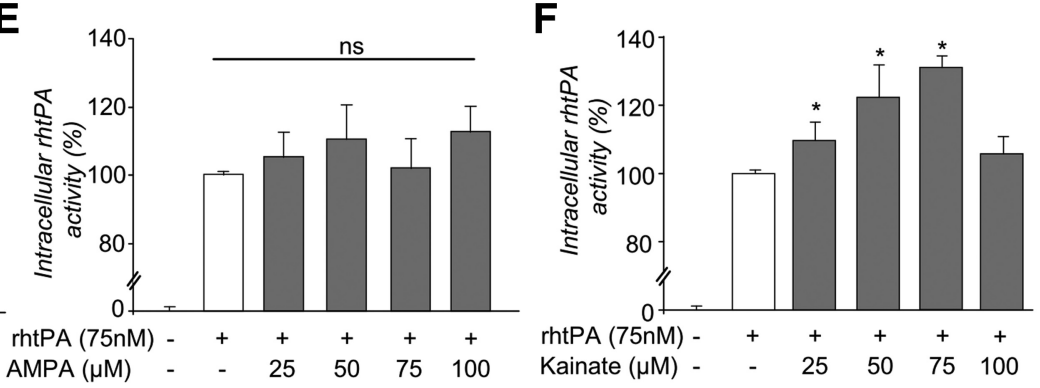

Figure 6. Glutamate increases the amount of tPA in astrocytes by acting on AMPA/kainate receptors. $A$, Astrocyte cultures were exposed for $15 \mathrm{~min}$ to rhtPA (75 $\mathrm{nm}$ ) alone or with glutamate (from 0.1-1 mm). Graphs show mean \pm SEM $(n=5)$ of quantification (enzymatic assay) of intracellular PA proteolytic activity (percentage of rhtPA condition). ${ }^{* *}$, Significantly ( $p<0.01$ ) different from tPA condition. ns: not significant. $\boldsymbol{B}-\boldsymbol{F}$, Astrocyte cultures were exposed for $15 \mathrm{~min}$ to the different agents as indicated. CNQX and NBQX are competitive antagonists of AMPA/kainate receptors. UBP 301 is a specific antagonist of kainate receptors. Graphs show mean \pm SEM $(n=3)$ of quantification (enzymatic assay) of intracellular tPA proteolytic activity (percentage of rhtPA condition). * $\#$, Significantly $(p<0.05)$ different from rhtPA or tPA plus glutamate condition. ns: not significant.

and subjected to electrophoresis in a SDS-PAGE (10\%). PA $^{555}$ was visualized using ImageQuant LAS 4000 camera.

\section{Intracortical injections of $t P A^{555}$}

Intracortical injections were performed in male Swiss mice. One microgram of $\mathrm{tPA}^{555}$ in a total volume of $1 \mu \mathrm{l}$ was injected with a microinjection pipette (internal diameter $0.32 \mathrm{~mm}$ and calibrated at $15 \mathrm{~mm} / \mu \mathrm{l}$; Hecht Assistent) into the left or right cortex at coordinates $0.5 \mathrm{~mm}$ posterior, $\pm 3 \mathrm{~mm}$ lateral, and $-0.8 \mathrm{~mm}$ ventral to the bregma. The needle was removed $5 \mathrm{~min}$ later. After $4 \mathrm{~h}$, mice were deeply anesthetized and perfused transcardially with $20 \mathrm{ml}$ of cold heparinized $\mathrm{NaCl} 0.9 \%$, followed by $2 \%$ paraformaldehyde and $0.2 \%$ picric acid in $150 \mathrm{ml}$ of $0.1 \mathrm{M}$ sodium phosphate buffer, $\mathrm{pH}$ 7.4. Brains were removed, washed in Veronal buffer containing 20\% sucrose, and frozen in Tissue-Tek (Miles Scientific). Coronal sections measuring $10 \mu \mathrm{m}$ each were incubated overnight at room temperature with a primary antibody mouse polyclonal anti-GFAP.

\section{Statistical analysis}

Results are expressed as mean \pm SEM. Statistical analyses were performed by the Kruskall-Wallis test, followed by post hoc comparisons with the Mann-Whitney test.

\section{Results}

\section{Astrocytes drive constitutive LRP-mediated endocytosis} of tPA

Astrocytes have been shown to endocytose and recycle synaptic glutamate. We wondered whether astrocytes may similarly regulate the amount of tPA. Uptake and accumulation of proteolytically active rhtPA was measured in cortical astrocyte monolayers by zymography (Figs. $1 A-C$ ) and the total amount of tPA protein was estimated by immunodetection using a specific anti-rhtPAantiserum (data not shown). The uptake occurred in a dosedependent (30-300 nM, Fig. $1 A, B)$ and time-dependent (from 5-120 min, Fig. 1C) manner. This astrocytic uptake of tPA was confirmed by observing the density of Alexa ${ }^{555}$-labeled tPA fluorescent staining in GFAP-positive astrocytes (Fig. $1 D, H$ ). This uptake was a rapid process, with $>80 \%$ of total tPA internalized after $2 \mathrm{~min}$ (Fig. $1 E$ ). These observations were further verified by flow cytometry analysis (Fig. $1 F$ ) for Alexa ${ }^{555}$-tPA-positive astrocytes. Altogether, these different approaches revealed a rapid uptake of exogenous tPA by astrocytes, occurring within the first minutes of exposure, with a rate of uptake of $\sim 11.3 \pm 1.1 \mathrm{pg}$ of tPA per minute per milligram of protein. Interestingly, after uptake, intracellular tPA remained proteolytically active (Fig. $1 A-$ $C)$. Uptake of tPA did not occur at $4^{\circ} \mathrm{C}$ (Fig. $\left.1 \mathrm{~F}-\mathrm{H}\right)$, indicating that it is an active process. As a control, astrocytes failed to uptake Alexa ${ }^{555}$-labeled albumin added at the same concentration (75 nM, Fig. $1 F-H)$.

As a first proof of endocytosis-mediated uptake of exogenous tPA, we investigated whether intracellular Alexa ${ }^{555}$-labeled tPA colocalizes with AP-2, a marker of clathrin-associated endocytotic vesicles (Schmid and McMahon, 2007) (Fig. 1I). Pretreatment of cells in the presence of DCV, to promote disassembly of clathrin cages, or dynasore, an inhibitor of dynamin, reduced the endocytosis of exogenous tPA, measured in terms of proteolytically active tPA in the cell monolayer $(-51.7 \pm 10.2 \%$ and $-90.5 \pm 2.4 \%$, respectively) (Fig. $1 \mathrm{~J}, \mathrm{~K}$ ) and by the density of Alexa ${ }^{555}$-tPA staining in GFAP-positive astrocytes (data not shown).

To investigate whether the uptake of tPA could also occur in vivo, we performed intracortical injections of Alexa ${ }^{555}$-tPA in mice. Brain sections were analyzed by confocal microscopy. In agreement with our in vitro observations, injected Alexa ${ }^{555}$-tPA was found inside GFAP-positive astrocytes (Fig. 2). These results show that astrocytes can uptake extracellular tPA in vivo.

LRP receptors, especially LRP1, are well known binding proteins for tPA (Zhang et al., 2009) involved in the hepatic clearance 


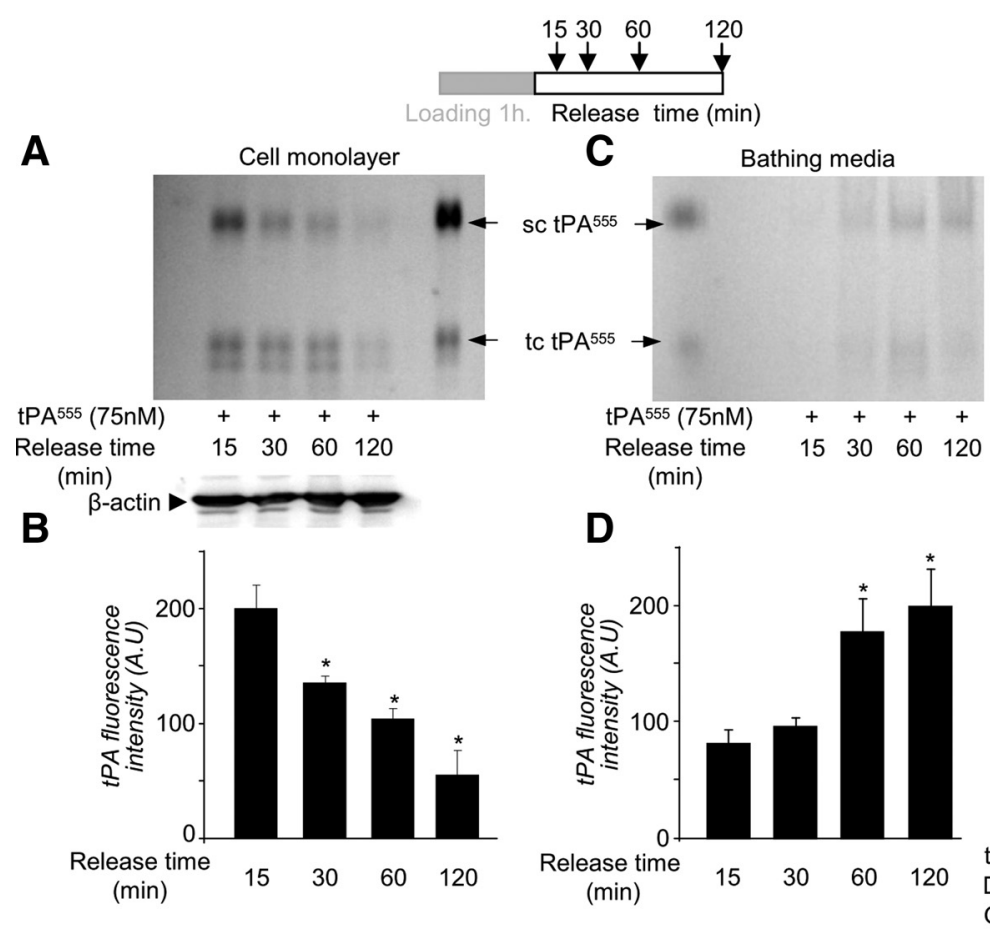

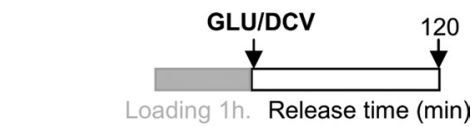
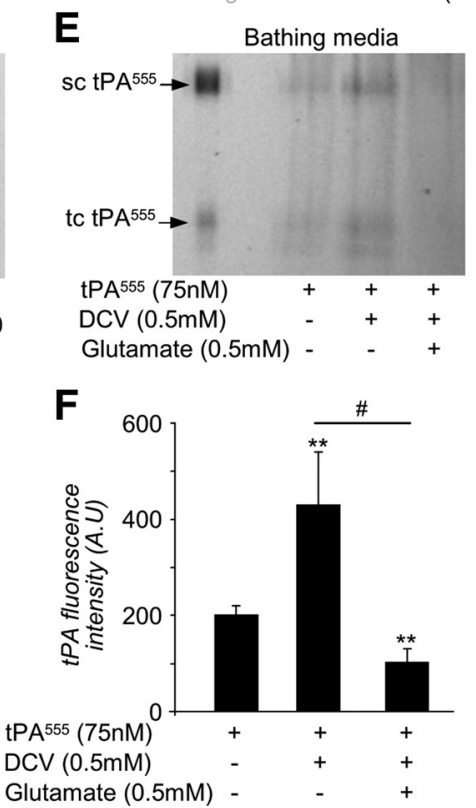

Figure 7. The recycling of tPA by astrocytes is dependent on extracellular glutamate. $A$, Astrocyte cultures were exposed to tPA 555 ( $300 \mathrm{nm)} \mathrm{for} 1 \mathrm{~h}$, then washed three times with PBS, and the bathing media were replaced with MS-glycine for 15-120 min. SDS-PAGE (representative of $n=3$ ) analysis of intracellular protein extracts shows tPA ${ }^{555}$ in cell monolayer of astrocytes. Western blot for $\beta$-actin was used as a loading control. sc-tPA and tc-tPA were detectable. $B$, Graph shows mean \pm SEM $(n=3)$ quantification of total tPA ${ }^{555}$ (sc-tPA plus tc-tPA) fluorescence in SDS-PAGE cell monolayer of astrocytes treated in $\boldsymbol{A}$. ${ }^{*}$, Significantly $(p<0.05)$ different from measurement 15 min after. $\boldsymbol{C}$, SDS-PAGE (representative of $n=3$ ) analysis of tPA ${ }^{555}$ from the bathing media corresponding to $\boldsymbol{A}$. $\boldsymbol{D}$, Graph shows mean $\pm \operatorname{SEM}(n=3)$ of quantification of total tPA ${ }^{555}$ (sc-tPA plus tc-tPA) fluorescence in SDS-PAGE of the bathing media shown in $\boldsymbol{C}$. ${ }^{*}$, Significantly $(p<0.05)$ different from measurement 15 min after release. $\boldsymbol{E}$, Astrocyte cultures were exposed to $\mathrm{PA}^{555}(300 \mathrm{~nm})$ for $1 \mathrm{~h}$, followed by three washes with PBS. Then the bathing media were replaced with MS-glycine with or without DCV $(0.5 \mathrm{~mm})$ for $10 \mathrm{~min}$. After three washes with PBS, the bathing media were replaced by MS-glycine with or without glutamate (0.5 mM). SDS-PAGE (representative of $n=6$ ) shows detection of $\mathrm{tPA}^{555}$ in the bathing media collected after $120 \mathrm{~min}$. $\boldsymbol{F}$, Graph shows mean \pm SEM $(n=6)$ of quantification of total tPA ${ }^{555}$ (sc-tPA plus tc-tPA) fluorescence in SDS-PAGE. ${ }^{* *}$, \#, Significantly $(p<0.01)$ different from tPA ${ }^{555}$ alone or PPA $^{555}$ plus DCV.

and in the blood-brain barrier passage of circulating tPA (Kuiper J et al., 1995; Benchenane et al., 2005). To investigate the potential role of LRP in the receptor-mediated endocytosis of tPA, we first assessed the expression of the isoforms of LRP receptors in astrocytes (LRP1, LRP1B, LRP2, and LRP4) by quantitative RT-PCR (Fig. 3A). LRP1 and LRP4 appeared to be the major isoforms expressed in astrocytes. In agreement with the colocalization of intracellular Alexa ${ }^{555}$-tPA with immunostaining for LRP1 (Fig. $3 B)$, RAP, an LRP receptor antagonist, reduced the astrocytic endocytosis of tPA (Fig. $3 C$ ). Interaction of tPA with LRP1 in astrocytes was confirmed by LRP1 coimmunoprecipitation of tPA (Fig. 3D). The rate of tPA uptake was comparable to that observed with the other LRP ligand, $\alpha 2$-macroglobulin (Fig. $3 E, F)$. To further study whether LRP1 and LRP4 could mediate tPA endocytosis, the expression of these receptors was knocked down using Stealth RNAi siRNA assay (Invitrogen) (Fig. 3G,H). Silencing of LRP1 or dual silencing of LRP1 and LRP4 (Fig. 3G) significantly reduced tPA endocytosis $(-66.2 \pm 5.1 \%$ and $-55.3 \pm 7.1 \%$, respectively, Fig. $3 H$ ), whereas silencing of LRP4 alone had no effect. These observations were confirmed by immunocytochemistry revealing an intracellular colocalization of Alexa ${ }^{555}$-tPA with LRP1 and AP-2 (Fig. 3I).

Altogether, these data indicate that the endocytosis of tPA by astrocytes mainly occurs via a receptor-mediated endocytosis involving at least LRP1.

To confirm the role of LRP in tPA endocytosis, we measured the endocytosis of a set of mutant forms of tPA to determine which domain or domains of tPA are essential for its uptake by astrocytes (Fig. 4A). Both single-chain tPA (sc-tPA) and twochain tPA (tc-tPA) were endocytosed by astrocytes (data not shown). Proteolytically inactive tPA (tPA-GGACK) (Fig. 4B) and tPA deleted for its epidermal growth factor-like domain $(\triangle E G F-$ tPA, Figs. $4 C, D$ ) were endocytosed by astrocytes similar to wildtype $\mathrm{tPA}$. In contrast, tPA deleted for its finger domain $(\Delta \mathrm{F}-\mathrm{tPA})$ was barely endocytosed $(-92 \pm 2.8 \%$ when compared with the wild-type tPA (Fig. 4C,D). Altogether, these data show that astrocytes drive constitutive endocytosis of tPA. This endocytosis involves the finger domain of TPA and the LRP1 subtype of LRP receptors, and occurs through a clathrin-dependent and dynamin-dependent mechanism.

\section{Intracellular trafficking of tPA in astrocytes}

Membrane fusion involved in the endocytotic and exocytotic pathways are mediated by the formation of a complex between a vesicular (v)-SNARE (often referred to as VAMP) with a target (t)-SNARE. Over the last decades, astrocytes were shown to present various types of vesicular organelles, which have the potency to fuse with the plasma membrane, thus releasing their content. VAMP-2 and VAMP-3 are present both on small vesicular organelles and large dense vesicles in astrocytes (Bezzi et al., 2004; Volterra and Meldolesi, 2005) and have been implicated in the release of glutamate (Araque et al., 1998), ATP (Coco et al., 2003), or D-serine (Mothet et al., 2005). TI-VAMP is also expressed in astrocytes and colocalizes with late endosomal/lysosomal vesicles (CD63-positive), which are able to undergo asynchronous exocytosis (Li et al., 2008). Thus, to further investigate the intracellular 
traffic of tPA, cultured astrocytes were transfected with a set of cDNA-encoding markers of several types of trafficking vesicles, including Rab5 (a small GTPase localized in early endosomes), VAMP-3, VAMP-4, CD63 (a late-endosomal marker), and TI-VAMP/VAMP-7, before incubation with Alexa ${ }^{555}$-tPA (Fig. 5).

Exogenously supplied Alexa ${ }^{555}$-tPA partially colocalized with intracellular markers, VAMP-3, VAMP-4, CD63, and TI-VAMP, and with Rab5 (Fig. 5A), indicating a complex traffic of tPA involving several endosomal compartments within astrocytes. Accordingly, transfection of a dominantnegative TI-VAMP fragment or an active form of the tetanus toxin $\left(\mathrm{TeNT}^{+}\right)$, which cleaves both VAMP-2 and VAMP-3, significantly reduced the amount of tPA accumulated in astrocytes (Fig. 5B,C) $(-68.4 \pm$ $6.4 \%)$. In contrast, transfection of an inactive TeNT $\left(\mathrm{TeNT}^{-}\right.$) or a dominantnegative form of Rab5 had no effect (Fig. $5 B, C)$. These results suggest that the endosomal system is required for tPA uptake by astrocytes.

\section{Extracellular glutamate promotes an} AMPA/kainate receptor-mediated accumulation of $\mathrm{TPA}$ in astrocytes by inhibiting the exocytotic recycling of tPA by astrocytes

tPA is considered a neuromodulator controlling glutamatergic signaling (Samson and Medcalf, 2006), while astrocytes have been reported to regulate the levels of glutamate available at the synapse (Pellerin and Magistretti, 1994). We investigated whether glutamate could influence the uptake and recycling of tPA by astrocytes.

When applied to cultured astrocytes, glutamate (0.1-1 mM) led to an increased intracellular concentration of tPA as measured either as tPA proteolytic activity (Fig. 6 A; $35.4 \pm 8.7 \%$ at $0.5 \mathrm{~mm}$ glutamate) or as amount of Alexa ${ }^{555}$-tPA (data not shown) in the cell monolayer. This effect was reduced by the coapplication of either CNQX (Fig. 6B) or NBQX (Fig. 6C), two competitive antagonists of AMPA/ kainate receptors. UBP 301, a specific antagonist of kainate receptors, prevented glutamate-induced increase in intracellular tPA (Fig. 6D). This was confirmed by the fact that presence of kainate alone $(25-75 \mu \mathrm{M})$ led to an increased intracellular concentration of $\mathrm{tPA}(30.7 \pm 3.7 \%)$, whereas AMPA $(25-100 \mu \mathrm{M})$ failed to do so (Figs. $6 E, F)$. Altogether, these data demonstrate that presence of extracellular glutamate leads to an increased accumulation of tPA in astrocytes through a mechanism involving astrocyte kainate receptors. In agreement with a kainatedependent mechanism, antagonists of NMDA receptors, mGluRI, mGluRII, and mGluRIII (respectively MK-801, AIDA, APICA, and CPPG) did not affect intracellular concentration of tPA in astrocytes (data not shown).

We postulated that the increased accumulation of exogenous tPA observed in the astrocyte monolayers in the presence of glutamate could be due to a differential sorting of tPA within the cell either through increased endocytosis or reduced exocytosis. To address this question, we performed follow-up experiments with Alexa ${ }^{555}$-labeled tPA (Fig. 7). Alexa ${ }^{555}$-labeled tPA was added in the bathing media for $1 \mathrm{~h}$ at $37^{\circ} \mathrm{C}$ ("loading" period), followed by extensive washing ("release" period). No cell death was detected during the time of these experiments (data not shown). tPA is secreted in sc-tPA and can be processed into tctPA by proteolytic action of plasmin or kallikrein (Rijken et al., 1982; Rajapakse et al., 2005). SDS-PAGE electrophoresis of proteins extracted from the cell layer revealed a decrease in both sc-tPA and tc-tPA with time during the release period (Fig. $7 A, B)$. Concomitantly, an increase in sc-tPA and tc-tPA was observed in the corresponding bathing media (Fig. 7C,D). These data suggest that tPA previously taken up can be released by astrocytes in a time-dependent manner. The sc-tPA/tc-tPA ratio did not change either in the cell monolayer or in the bathing media, indicating that no processing of sc-tPA into tc-tPA occurred during this recycling (data not shown). Then, we tested whether or not extracellular glutamate affects $t$ PA recycling (Fig. $7 E, F)$. DCV was applied to prevent secondary endocytosis so that only the release of tPA was observed. DCV alone, added during the release period, led to an accumulation of Alexa ${ }^{555}-\mathrm{tPA}$ 
A

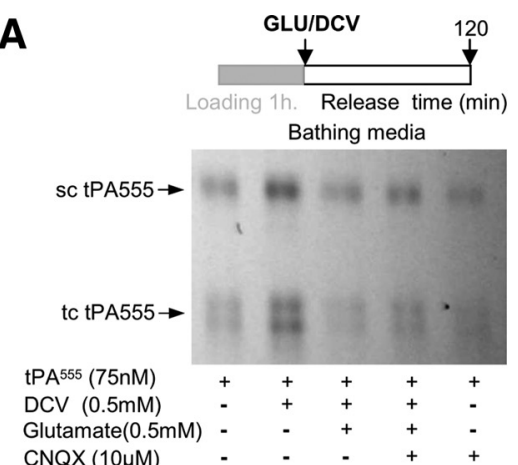

B

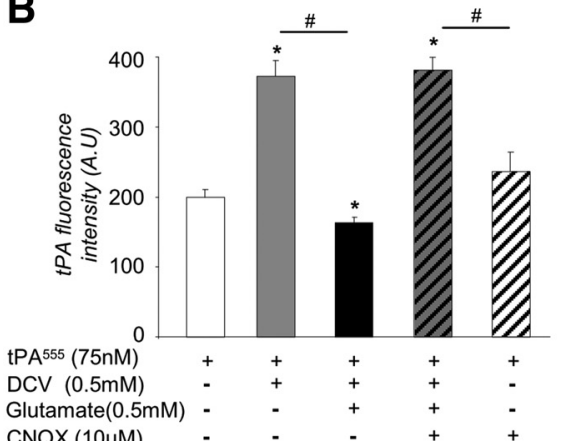

D

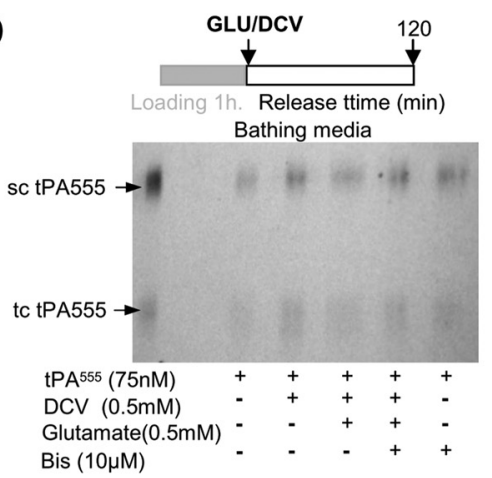

$\mathbf{E}$

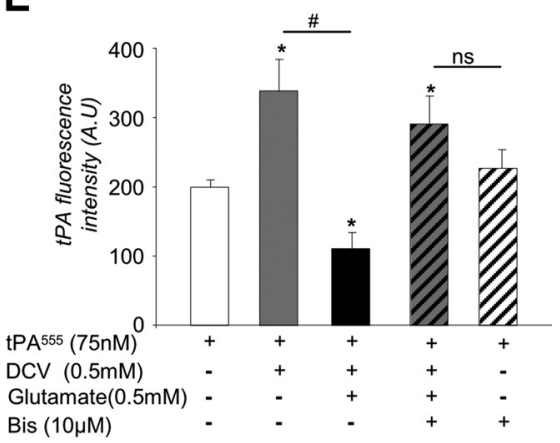

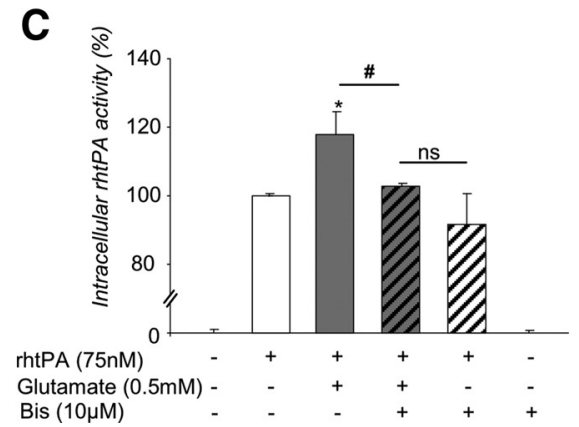

Figure 9. Astrocytic tPA recycling involves a PKC-dependent signaling pathway. $A$, Astrocyte cultures were exposed to tPA 555 $(300 \mathrm{~nm})$ for $1 \mathrm{~h}$, then washed with PBS three times. Then, for $10 \mathrm{~min}$, cultures were placed in a bathing media of pure MS-glycine or MS-glycine containing DCV $(0.5 \mathrm{~mm})$. The cells were washed with PBS three times, and the bathing media were (1) replaced by pure MS-glycine, (2) replaced by MS-glycine containing glutamate $(0.5 \mathrm{mM})$ with CNQX (10 $\mu \mathrm{M})$, or (3) replaced by MS-glycine containing glutamate $\left(0.5 \mathrm{~mm}\right.$ ) without CNQX. SDS-PAGE (representative of $n=3$ ) analysis of $\mathrm{PPA}^{555}$ in the bathing media collected after $120 \mathrm{~min}$. $\boldsymbol{B}$, Graph shows mean \pm SEM $(n=3)$ of quantification of total tPA ${ }^{555}$ (sc-tPA plus tc-tPA) fluorescence in SDS-PAGE. * \#, Significantly $(p<0.05)$ different from tPA ${ }^{555}$ alone or PA $^{555}$ plus DCV. C, Astrocyte cultures were exposed for 15 min to $\mathrm{PPA}(75 \mathrm{~nm})$ alone, $\mathrm{tPA}(75 \mathrm{nM})$ with glutamate $(0.5 \mathrm{~mm}), \mathrm{PA}(75 \mathrm{~nm})$ with bis $(10 \mu \mathrm{M})$, or $\mathrm{PPA}(75 \mathrm{~nm})$ with both glutamate $(0.5 \mathrm{mM})$ and bis $(10 \mu \mathrm{M})$. Graph shows mean \pm SEM $(n=3)$ of quantification (enzymatic assay) of intracellular tPA proteolytic activity (percentage of rhtPA condition). ${ }^{*}$, \#, Significantly $(p<0.05)$ different from rhtPA or rhtPA plus glutamate. ns: not significant. D, Astrocyte cultures were exposed to $\mathrm{PA}^{555}(300 \mathrm{~nm})$ for $1 \mathrm{~h}$, then washed with PBS three times. The bathing media were replaced with either pure MS-glycine or MS-glycine containing DCV $(0.5 \mathrm{~mm})$ for $10 \mathrm{~min}$. The cells were washed with PBS three times, and the bathing media were replaced by either pure MS-glycine or MS-glycine containing glutamate $(0.5 \mathrm{~mm})$ with or without BIS $(10 \mu \mathrm{M})$. SDS-PAGE (representative of $n=4$ ) analysis of $\mathrm{PAA}^{555}$ in the bathing media collected after $120 \mathrm{~min}$. $E$, Graph shows mean \pm SEM $(n=4)$ of quantification of total tPA ${ }^{555}$ (sc-tPA plus tc-tPA) fluorescence in SDS-PAGE. *, \#, Significantly $(p<0.05)$ different from $\mathrm{PA}^{555}$ alone or $\mathrm{PA}^{555}$ plus DCV.

in the bathing media (Fig. 7E). Addition of glutamate reduced the accumulation of Alexa ${ }^{555}$-tPA $(-91.2 \pm 4.5 \%)($ Fig. $7 F)$ in the bathing media. These data show that astrocytes are able to release the tPA previously taken up, and that glutamate inhibits this release.

Part of internalized tPA was driven to degradation pathway, as shown by colocalization with the lysosome marker LysoTracker (Fig. $8 \mathrm{~A}$ ) and estimation of the relative levels of internalized, recycled, and degraded Alexa ${ }^{555}$-tPA $(59 \pm 5.9 \%, 26.7 \pm 3.4 \%$, and $14.3 \pm$ $2.7 \%$, respectively, after 1 h; Fig. $8 \mathrm{~B}$ ).

To further elucidate the mechanism of tPA recycling, uptake of $\mathrm{tPA}$ by astrocytes was monitored in the presence of AMPAreceptor and kainate-receptor antagonists (Fig. 9A,B). In agreement with the above data (Fig. 6), CNQX, an AMPA/kainate receptor antagonist, prevented the inhibitory effect of glutamate on $\mathrm{tPA}$ recycling.

Kainate receptors were previously reported to mediate $\mathrm{PKC}$ activation (Rodríguez-Moreno and Lerma, 1998), and PKC was shown to regulate exocytosis in astrocytes (Yasuda et al., 2011). Hence we tested whether this pathway controls the astrocytic recycling of tPA. The PKC inhibitor bis $(10 \mu \mathrm{M})$ led to a reduced accumulation of intracellular tPA when applied in the presence of glutamate during the loading procedure $(-14.4 \pm 6.0 \%$, Fig. 9C) and a reduced recycling of tPA when applied in the presence of glutamate during the release period $(-13.1 \pm 3.5 \%$, Fig. $9 D, E)$. Together, these data argue for a mechanism of tPA recycling involving kainate receptor-mediated, PKC-dependent signaling.

\section{Astrocytes regulate the amount of extracellular tPA and the influence of tPA on NMDA-induced excitotoxicity in neurons}

To investigate the physiological relevance of the trafficking of tPA in astrocytes, we used a model of mixed cultures of cortical neurons and astrocytes, in which tPA-lumio was expressed specifically in neurons, through the transfection of an expression vector driven by the synapsin promoter. Thus, we were able to monitor and quantify (1) the release of tPA-lumio by challenged neurons and (2) the accumulation of the neuron-derived tPA-lumio in astrocytes by time-lapse confocal imaging (Fig. 10A,B). Depolarization was induced by the coapplication of biccuculine and 4-AP $\left(\mathrm{a} \mathrm{GABA}_{\mathrm{A}}\right.$ inhibitor and a blocker of the voltage-dependent channels) so that the neurons released tPA-lumio $(-34.7 \pm$ $3.3 \%$ of intracellular tPA-lumio in neuronal cells after $150 \mathrm{~min}$, Fig. 10C). This tPA-lumio was then endocytosed by neighboring astrocytes in a timedependent manner (40.2 $\pm 3.8 \%$ after $150 \mathrm{~min}$, Fig. $10 \mathrm{D}, E$, $)$. Silencing of LRP1 prevented the accumulation of neuron-derived tPA-lumio in the neighboring astrocytes, without affecting the release of tPA-lumio by neuronal cells (Fig. 10C-E). The accumulation of neuron-derived tPA-lumio was also prevented by the coapplication of the AMPA/kainate receptor antagonist, CNQX, applied $1 \mathrm{~h}$ after the neuronal depolarization $(-47.2 \pm 3.6 \%$, Fig. $10 F-H)$. 


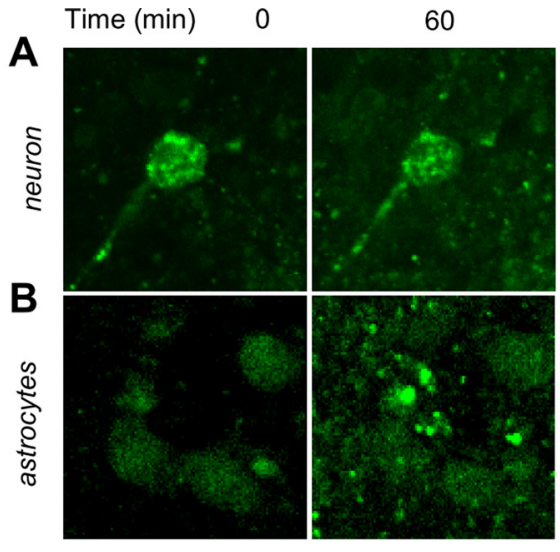

C
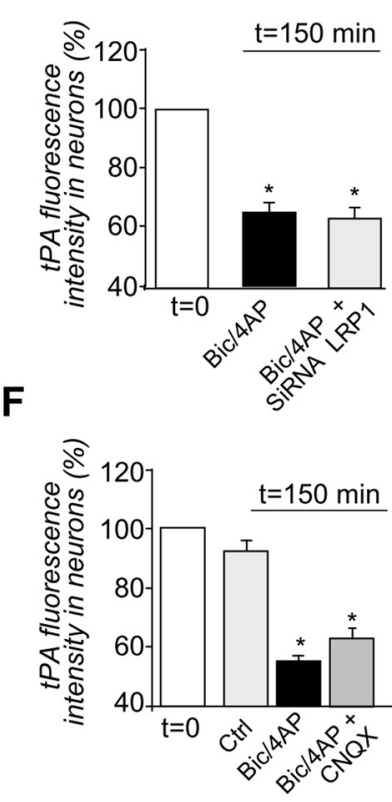

D

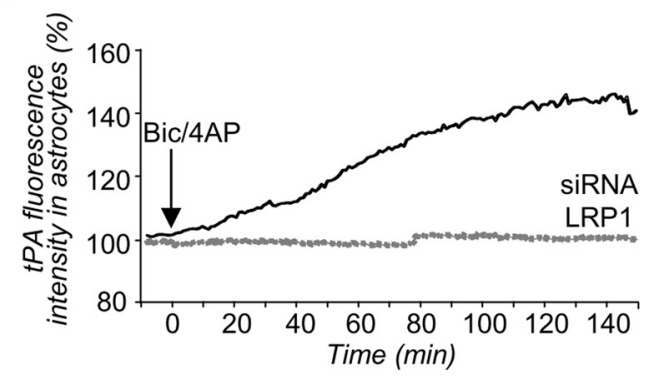

G

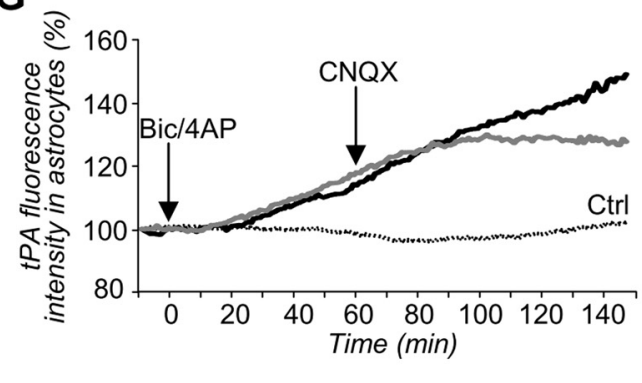

140

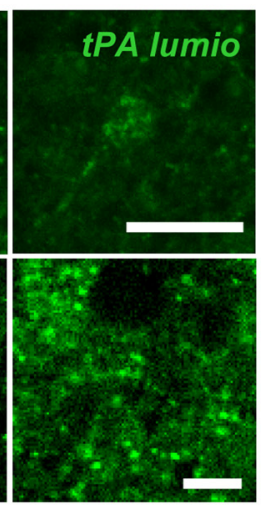

E
H

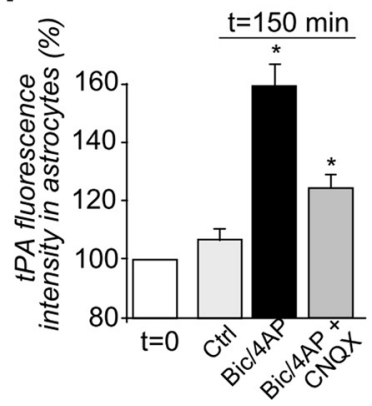

Figure 10. Astrocytes recapture of neuron-derived tPA. A, B, Mixed cultures of neurons and astrocytes were transfected at 12 DIV with pSyn-tPA-lumio vector and were subjected to depolarization, at $t=0 \mathrm{~min}$, with biccuculine $(50 \mu \mathrm{m})$ plus 4-AP $(2.5 \mathrm{~mm})$. Photomicrographs show live cell confocal imaging (representative image of $n=3)$ of tPA-lumio (in green) in neurons $(A)$ or astrocytes $(\boldsymbol{B})$ at 0,60 , and $140 \mathrm{~min}$ after depolarization. Scale bars, $30 \mu \mathrm{m}$. $\boldsymbol{C}$, Mixed cultures were transfected at 12 DIV with pSyn-tPA-lumio with or without LRP1 siRNA vectors and were subjected to depolarization at $t=0 \mathrm{~min}$ with biccuculine $(50 \mu \mathrm{m})$ plus 4-AP $(2.5 \mathrm{~mm})$. Graphs show mean \pm SEM $(n=3)$ of quantification of tPA-lumio fluorescence in neurons. $D, E$, Accumulation of tPA-lumio fluorescence in astrocytes from the beginning $(t=0 \mathrm{~min})$ to the end $(t=150 \mathrm{~min})$ of the experiment. ${ }^{*}$, Significantly $(p<0.05)$ different from $t=0 \mathrm{~min}$. Black line, Control conditions. Dotted line, LRP siRNA condition. $\boldsymbol{F}$, Mixed cultures transfected (at 12 DIV) with pSyn-tPA-lumio were subjected or not (control) to depolarization, at $t=0$ min, with biccuculine (50 $\mu \mathrm{M}$ ) plus 4 -AP $(2.5 \mathrm{~mm})$. CNQX or vehicle were added at $t=60 \mathrm{~min}$. Graphs show mean $\pm \mathrm{SEM}(n=3)$ of the quantifications of tPA-lumio fluorescence in neurons. $\mathbf{G}, \boldsymbol{H}$, Accumulation of tPA-lumio fluorescence by astrocytes from the beginning $(t=0 \mathrm{~min})$ to the end $(t=150 \mathrm{~min}) .{ }^{*}$, Significantly $(p<0.05)$ different from condition at $t=0 \mathrm{~min}$. Gray line, CNQX treatment. Black line, Vehicle treatment. Dotted line, Control (not depolarized).

tPA was previously reported to promote NMDA receptor signaling and consequent excitotoxicity (Nicole et al., 2001). To study whether the astrocytic uptake/recycling of tPA could influence its action on neurons, we compared the ability of tPA to promote NMDA receptor-mediated neuronal death in pure cultures of neurons with that in mixed cultures of neurons and astrocytes (Fig. 11). Although application of NMDA alone led to the death of $56.8 \pm 3.4 \%$ of neurons in pure cultures, this effect was reduced in cultures containing astrocytes $(33.8 \pm 6.1 \%$ ) (Fig. $11 A, B)$. Furthermore, addition of tPA promoted NMDAinduced neuronal death in pure neuronal cultures, but not in mixed cultures of neurons and astrocytes (Fig. 11 $A, B$ ). Hence, we hypothesized that the lack of proneurotoxicity of $\mathrm{PA}$ in mixed cultures may be explained by the capture of exogenous tPA by astrocytes. To confirm this hypothesis, experiments were performed with tPA deleted for its finger domain, which is resistant to astrocytic endocytosis (Fig. 4). Consistent with the hypothesis, this modified tPA molecule displayed proneurotoxic effects both in pure neuronal cultures and in mixed cultures of neurons and astrocytes (Fig. $11 C, D, 25.3 \pm 6.7 \%$ and $31.1 \pm 2.9 \%$, respectively). These results show that the astrocytic uptake of tPA prevents tPA from promoting NMDA receptor-mediated effects in neurons. Altogether, these results indicate that astrocytes are able to capture extracellular $\mathrm{PAA}$, and to protect neuronal cells from tPA-induced cell death, by preventing recycling of tPA in the extracellular space when extracellular glutamate is present.

\section{Discussion}

The present study reveals a new modality of cross talk between neurons and astrocytes. On the one hand, astrocytes mediate a constitutive LRP-dependent endocytosis of neuron-derived tPA; on the other hand, astrocytes drive an exocytotic recycling of tPA, 

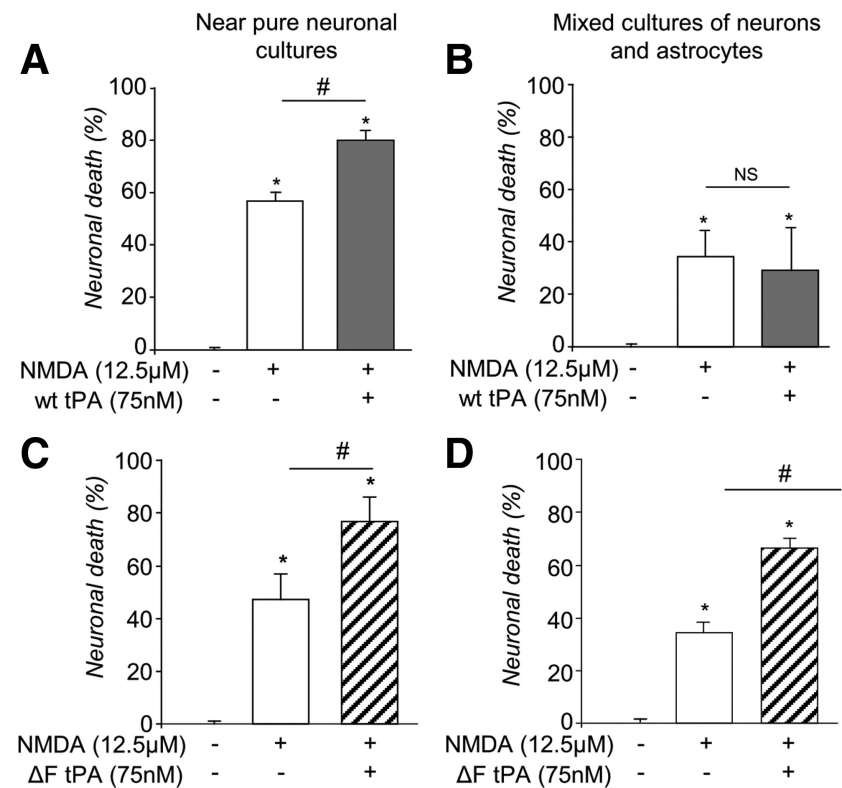

Figure 11. tPA recapture in astrocytes abolishes the effects of tPA on NMDA-mediated neuronal death. $\boldsymbol{A}-\boldsymbol{D}$, Graphs show neuronal death (percentage of control) mean $\pm \operatorname{SEM}(n=3)$ of pure cultures of neurons (12-14 DIV) $(\boldsymbol{A})$ or mixed cultures of neurons and astrocytes (12-14 DIV) $(\boldsymbol{B})$ exposed for $24 \mathrm{~h}$ to NMDA $(12.5 \mu \mathrm{M})$ with or without $\mathrm{PAA}(300 \mathrm{nM})$ or pure cultures of neurons (12-14 DIV) (C) or mixed cultures of neurons and astrocytes $(\boldsymbol{D})$ exposed for $24 \mathrm{~h}$ to $\mathrm{NMDA}(12.5 \mu \mathrm{M})$ with or without $\Delta \mathrm{F} \mathrm{PA}(300 \mathrm{~nm})$. LDH released into the bathing medium was assessed $24 \mathrm{~h}$ after the beginning of the excitotoxic exposure. ${ }^{*}$, \#, Significantly $(p<0.05)$ different from, respectively, control or NMDA treatment. ns: not significant.

inhibited by extracellular glutamate via a kainate receptormediated, PKC-dependent signaling pathway. Kainate receptors, thus acting as sensors of extracellular glutamate, enable astrocytes to regulate the extracellular quantity of tPA, so that as the concentration of glutamate rises, the quantity of tPA falls. By this mechanism, astrocytes can buffer extracellular tPA and thus reduce the effects of TPA on NMDA receptor-mediated events in neurons. This is the first demonstration that tPA, previously shown to play critical functions in the CNS as a neuromodulator (Fernández-Monreal et al., 2004; Samsom and Medcalf, 2006), also acts as a gliotransmitter.

We provide here the demonstration of a mechanism through which LRP receptors of astrocytes permit endocytosis of tPA and thus modulate the amount of extracellular neuron-derived tPA. We proved that the LRP1 subtype is involved in the astrocytic uptake of tPA. These data complement previous findings showing that the LRPs, especially LRP1, are endocytic receptors that recognize a wide range of ligands, including protease and protease inhibitor complexes, such as tPA (Lillis et al., 2008). In addition to their involvement in the hepatic clearance of circulating tPA (Kuiper et al., 1995), LRPs were also reported to modulate tPA-dependent cell migration (Cao et al., 2006), microglial activation (Zhang. et al., 2009), NMDA receptor signaling (Sheng et al., 2008), and integrity of the blood-brain barrier (Yepes et al., 2003). Previous studies, including some from our group, have demonstrated that LRP receptors can mediate transcytosis of tPA across the endothelial cells (Benchenane et al., 2005), leading to the notion that LRP receptors are possible regulators of the uptake of tPA in astrocytes (Fernández-Monreal et al., 2004).

Based on the data presented here, we know that extracellular glutamate activates kainate receptors in astrocytes, and is able to influence the levels of extracellular tPA. This mechanism is reminiscent of the classical neuron-astrocyte coupling in which glu- tamate, released during synaptic transmission, is taken up by astrocytes and converted to glutamine before being transported back to neurons and recycled into glutamate (Pellerin and Magistretti, 1994). Endogenous levels of glutamate, produced by depolarized neurons, appear to be sufficient to modulate tPA recycling, at least in our in vitro system, as the application of glutamate antagonist (Fig. 9G) slows down the accumulation of neuron-derived tPA in astrocytes. Our data strongly indicate a key role played by kainate receptors in inhibition of exocytosis of tPA. Nevertheless, considering the possible pharmacological overlap of AMPA and kainate receptor antagonists, participation of AMPA receptors cannot be totally ruled out.

Our study also demonstrates that inhibition of tPA recycling by kainate receptor activation involves a PKC-dependent signaling pathway. Kainate receptors are known to drive, in addition to their ionotropic functions, metabotropic functions leading to PKC activation in neurons (Rodríguez-Moreno and Lerma, 1998). This kainate receptor-dependent PKC activation was reported to inhibit neuronal release of GABA (Rodríguez-Moreno and Lerma, 1998). In parallel, PKC was shown to phosphorylate the $25 \mathrm{kDa}$ synaptosome-associated protein (SNAP-25), a SNARE protein. Similar mechanisms may regulate tPA recycling in astrocytes: PKC activation was reported to inhibit $\mathrm{Ca}^{2+}$ dependent exocytosis of proteins through the phosphorylation of SNAP-23, an astrocytic equivalent of neuronal SNAP-25 (Yasuda et al., 2011). In the present study, inhibition of both kainate receptors and PKC signaling reversed glutamate-induced inhibition of tPA exocytosis. These results thus suggest that kainate receptor activation by glutamate would lead to PKC activation and subsequent reduction of tPA exocytosis. Further studies may reveal the role of SNAP-23 phosphorylation in these processes.

We demonstrate that astrocytes can modulate the effective concentration of extracellular tPA, thereby influencing NMDA receptor signaling and subsequent excitotoxic neuronal death. This implies that, at least in vitro, the amount of tPA cleared by astrocytes is sufficient to potentiate NMDA-induced excitotoxicity. The amount of tPA available in the extracellular space may also be critical in a number of other tPA-related brain functions and dysfunctions. For example, during development, tPA has been shown to promote synaptic outgrowth or neuronal migration, probably by facilitating degradation of extracellular matrix during axon elongation (Seeds et al., 1997). In the adult, the tPA gene is an immediate-early gene, is induced by neuronal activity (Qian et al., 1993) and tPA action, is considered neuromodulatory (Samson and Medcalf, 2006), and participates in the control of NMDAR-dependent LTP (Zhuo et al., 2000). It would be interesting to see whether astrocytic $\mathrm{AA}$ recycling could also influence the multiple effects of tPA in the brain, such as LRPdependent signaling (Lillis et al., 2008), activation of PDGF signaling (Su et al., 2008), microglial activation (Siao and Tsirka, 2002), blood-brain barrier leakage (Yepes et al., 2003), activation of the EGF pathway in oligodendrocytes (Correa et al., 2011), or plasmin-dependent activation of pro-BDNF into mature BDNF (Pang et al., 2004). Interestingly, although pro-BDNF was reported to be cleared by astrocytes through interaction with the pan-neurotrophin receptor p75-mediated clathrin-dependent endocytosis, intracellular pro-BDNF is also routed into a fast recycling pathway controlled by glutamatergic signaling (Bergami et al., 2008).

In this study, we showed that, following clathrin-dependent and dynamin-dependent endocytosis, tPA follows a complex sorting in astrocytes. Intracellular tPA colocalized with VAMP-3, VAMP-4, CD63, TI-VAMP/VAMP-7, and Rab5. VAMP-3 is a 
$\mathrm{v}$-SNARE of the early endosome, whereas VAMP-4 is a v-SNARE on endosome and trans-Golgi networks. VAMP-2 mediates the exocytosis of synaptic vesicles in neurons and neuroendocrine cells (Schiavo et al., 1992). Cellubrevin/VAMP-3 is the nonneuronal homolog of synaptobrevins (McMahon et al., 1993), a class of molecules involved in exocytosis. TI-VAMP localizes to the trans-Golgi network (Danglot et al., 2010) and to late endosomes and lysosomes (Chaineau et al., 2009). The absence of effect of the transfection of a dominant-negative form of Rab5 is likely due to redundancy of small GTPases involved in early endocytosis (Pavlos and Jahn, 2011). Our results thus favor a mechanism of endocytosis, including early and late endosomes, possibly a partial fusion with lysosomes and a recycling pathway by exocytosis. Although we showed here that part of the tPA taken up by astrocytes can be recycled and released, further studies would help to further characterize the fate of tPA after its uptake. In particular, the data presented here suggest that some of this tPA may be degraded. Also, the possibility that part of tPA may be rendered proteolytically inactivate should be addressed.

Astrocytes release a number of neuromodulators/neurotransmitters (Araque et al., 1998; Bender and Norenberg, 2000; Mothet et al., 2005), termed gliotransmitters. The term gliotransmitter generally refers to small molecules, such as ATP, adenosine, glutamate, GABA, or D-serine. The present study proposes to include tPA in the list of gliotransmitters. IPA is a critical element in shaping the complex and fundamental roles of astrocytes in the control of the brain homeostasis. The demonstration shown here of tPA sorting between neurons and astrocytes suggests critical roles for tPA in diseases of the CNS, such as ischemic brain injuries, head and spinal trauma, and multiple sclerosis (Gveric et al., 2005). Also, uptake of tPA by astrocytes may influence physiological processes that involve tPA, such as those related to learning and memory (Obiang et al., 2011), anxiety (Pawlak et al., 2003), and addiction (Nagai et al., 2004). This study opens up new avenues to further investigate the influence of astrocyte traffic of tPA on these physiological and pathological processes within the brain.

\section{References}

Araque A, Parpura V, Sanzgiri RP, Haydon PG (1998) Glutamatedependent astrocyte modulation of synaptic transmission between cultured hippocampal neurons. Eur J Neurosci 10:2129-2142.

Araque A, Li N, Doyle RT, Haydon PG (2000) SNARE protein-dependent glutamate release from astrocytes. J Neurosci 20:666-673.

Baranes D, Lederfein D, Huang YY, Chen M, Bailey CH, Kandel ER (1998) Tissue plasminogen activator contributes to the late phase of LTP and to synaptic growth in the hippocampal mossy fiber pathway. Neuron 21:813-825.

Benchenane K, Berezowski V, Ali C, Fernández-Monreal M, López-Atalaya JP, Brillault J, Chuquet J, Nouvelot A, MacKenzie ET, Bu G, Cecchelli R, Touzani O, Vivien D (2005) Tissue-type plasminogen activator crosses the intact blood-brain barrier by low-density lipoprotein receptorrelated protein-mediated transcytosis. Circulation 111:2241-2249.

Bender AS, Norenberg MD (2000) Effect of ammonia on GABA uptake and release in cultured astrocytes. Neurochem Int 36:389-395.

Bergami M, Santi S, Formaggio E, Cagnoli C, Verderio C, Blum R, Berninger B, Matteoli M, Canossa M (2008) Uptake and recycling of pro-BDNF for transmitter-induced secretion by cortical astrocytes. J Cell Biol 183:213-221.

Bezzi P, Gundersen V, Galbete JL, Seifert G, Steinhäuser C, Pilati E, Volterra A (2004) Astrocytes contain a vesicular compartment that is competent for regulated exocytosis of glutamate. Nat Neurosci 7:613-620.

Cao C, Lawrence DA, Li Y, Von Arnim CA, Herz J, Su EJ, Makarova A, Hyman BT, Strickland DK, Zhang L (2006) Endocytic receptor LRP together with tPA and PAI-1 coordinates Mac-1-dependent macrophage migration. EMBO J 25:1860-1870.

Chaineau M, Danglot L, Galli T (2009) Multiple roles of the vesicular-
SNARE TI-VAMP in post-Golgi and endosomal trafficking. FEBS Lett 583:3817-3826.

Coco S, Calegari F, Pravettoni E, Pozzi D, Taverna E, Rosa P, Matteoli M, Verderio C (2003) Storage and release of ATP from astrocytes in culture. J Biol Chem 278:1354-1362.

Correa F, Gauberti M, Parcq J, Macrez R, Hommet Y, Obiang P, Hernangómez M, Montagne A, Liot G, Guaza C, Maubert E, Ali C, Vivien D, Docagne F (2011) Tissue plasminogen activator prevents white matter damage following stroke. J Exp Med 208:1229-1242.

Danglot L, Chaineau M, Dahan M, Gendron MC, Boggetto N, Perez F, Galli T (2010) Role of TI-VAMP and CD82 in EGFR cell-surface dynamics and signaling. J Cell Sci 123:723-735.

Fernández-Monreal M, López-Atalaya JP, Benchenane K, Léveillé F, Cacquevel M, Plawinski L, MacKenzie ET, Bu G, Buisson A, Vivien D (2004) Is tissue-type plasminogen activator a neuromodulator? Mol Cell Neurosci 25:594-601.

Galli T, Haucke V (2004) Cycling of synaptic vesicles: how far? How fast! Sci STKE 2004:re19.

Gveric D, Herrera BM, Cuzner ML (2005) tPA receptors and the fibrinolytic response in multiple sclerosis lesions. Am J Pathol 166:1143-1151.

Haydon PG, Carmignoto G (2006) Astrocyte control of synaptic transmission and neurovascular coupling. Physiol Rev 86:1009-1031.

Kuiper J, Otter M, Voorschuur AH, van Zonneveld AJ, Rijken DC, van Berkel TJ (1995) Characterization of the interaction of a complex of tissue-type plasminogen activator and plasminogen activator inhibitor type 1 with rat liver cells. Thromb Haemost 74:1298-1304.

Li D, Ropert N, Koulakoff A, Giaume C, Oheim M (2008) Lysosomes are the major vesicular compartment undergoing $\mathrm{Ca}^{2+}$-regulated exocytosis from cortical astrocytes. J Neurosci 28:7648-7658.

Lillis AP, Van Duyn LB, Murphy-Ullrich JE, Strickland DK (2008) LDL receptor-related protein 1: unique tissue-specific functions revealed by selective gene knockout studies. Physiol Rev 88:887-918.

Mallard F, Tang BL, Galli T, Tenza D, Saint-Pol A, Yue X, Antony C, Hong W, Goud B, Johannes L (2002) Early/recycling endosomes-to-TGN transport involves two SNARE complexes and a Rab6 isoform. J Cell Biol 156:653-664.

Martinez-Arca S, Alberts P, Zahraoui A, Louvard D, Galli T (2000) Role of tetanus neurotoxin insensitive vesicle-associated membrane protein (TIVAMP) in vesicular transport mediating neurite outgrowth. J Cell Biol 149:889-900.

McMahon HT, Ushkaryov YA, Edelmann L, Link E, Binz T, Niemann H, Jahn R, Südhof TC (1993) Cellubrevin is a ubiquitous tetanus-toxin substrate homologous to a putative synaptic vesicle fusion protein. Nature 364:346-349.

Mothet JP, Pollegioni L, Ouanounou G, Martineau M, Fossier P, Baux G (2005) Glutamate receptor activation triggers a calcium-dependent and SNARE protein-dependent release of the gliotransmitter D-serine. Proc Natl Acad Sci U S A 102:5606-5611.

Nagai T, Yamada K, Yoshimura M, Ishikawa K, Miyamoto Y, Hashimoto K, Noda Y, Nitta A, Nabeshima T (2004) The tissue plasminogen activatorplasmin system participates in the rewarding effect of morphine by regulating dopamine release. Proc Natl Acad Sci U S A 101:3650-3655.

Nicole O, Docagne F, Ali C, Margaill I, Carmeliet P, MacKenzie ET, Vivien D, Buisson A (2001) The proteolytic activity of tissue-plasminogen activator enhances NMDA receptor-mediated signaling. Nat Med 7:59-64.

Obiang P, Maubert E, Bardou I, Nicole O, Launay S, Bezin L, Vivien D, Agin V (2011) Enriched housing reverses age-associated impairment of cognitive functions and tPA-dependent maturation of BDNF. Neurobiol Learn Mem 96:121-129.

Pang PT, Teng HK, Zaitsev E, Woo NT, Sakata K, Zhen S, Teng KK, Yung WH, Hempstead BL, Lu B (2004) Cleavage of proBDNF by tPA/plasmin is essential for long-term hippocampal plasticity. Science 306:487-491.

Pavlos NJ, Jahn R (2011) Distinct yet overlapping roles of Rab GTPases on synaptic vesicles. Small Gtpases 2:77-81.

Pawlak R, Magarinos AM, Melchor J, McEwen B, Strickland S (2003) Tissue plasminogen activator in the amygdala is critical for stress-induced anxiety-like behavior. Nat Neurosci 6:168-174.

Pellerin L, Magistretti PJ (1994) Glutamate uptake into astrocytes stimulates aerobic glycolysis: a mechanism coupling neuronal activity to glucose utilization. Proc Natl Acad Sci U S A 91:10625-10629.

Qian Z, Gilbert ME, Colicos MA, Kandel ER, Kuhl D (1993) Tissue- 
plasminogen activator is induced as an immediate-early gene during seizure, kindling and long-term potentiation. Nature 361:453-457.

Rajapakse S, Ogiwara K, Takano N, Moriyama A, Takahashi T (2005) Biochemical characterization of human kallikrein 8 and its possible involvement in the degradation of extracellular matrix proteins. FEBS Lett 579:6879-6884

Rijken DC, Hoylaerts M, Collen D (1982) Fibrinolytic properties of onechain and two-chain human extrinsic (tissue-type) plasminogen activator. J Biol Chem 257:2920-2925.

Rodríguez-Moreno A, Lerma J (1998) Kainate receptor modulation of GABA release involves a metabotropic function. Neuron 20:1211-1218.

Samson AL, Medcalf RL (2006) Tissue-type plasminogen activator: a multifaceted modulator of neurotransmission and synaptic plasticity. Neuron 50:673-678.

Samson AL, Nevin ST, Croucher D, Niego B, Daniel PB, Weiss TW, Moreno E, Monard D, Lawrence DA, Medcalf RL (2008) Tissue-type plasminogen activator requires a co-receptor to enhance NMDA receptor function. J Neurochem 107:1091-1101.

Schiavo G, Benfenati F, Poulain B, Rossetto O, Polverino de Laureto P, DasGupta BR, Montecucco C (1992) Tetanus and botulinum-B neurotoxins block neurotransmitter release by proteolytic cleavage of synaptobrevin. Nature 359:832-835.

Schmid EM, McMahon HT (2007) Integrating molecular and network biology to decode endocytosis. Nature 448:883-888.

Seeds NW, Siconolfi LB, Haffke SP (1997) Neuronal extracellular proteases facilitate cell migration, axonal growth, and pathfinding. Cell Tissue Res 290:367-370.

Sheehan JJ, Zhou C, Gravanis I, Rogove AD, Wu YP, Bogenhagen DF, Tsirka SE (2007) Proteolytic activation of monocyte chemoattractant protein-1 by plasmin underlies excitotoxic neurodegeneration in mice. J Neurosci 27:1738-1745.

Sheng Z, Prorok M, Brown BE, Castellino FJ (2008) N-methyl-D-aspartate receptor inhibition by an apolipoprotein E-derived peptide relies on low- density lipoprotein receptor-associated protein. Neuropharmacology 55:204-214.

Siao CJ, Tsirka SE (2002) Tissue plasminogen activator mediates microglial activation via its finger domain through annexin II. J Neurosci 22:3352-3358.

Su EJ, Fredriksson L, Geyer M, Folestad E, Cale J, Andrae J, Gao Y, Pietras K, Mann K, Yepes M, Strickland DK, Betsholtz C, Eriksson U, Lawrence DA (2008) Activation of PDGF-CC by tissue plasminogen activator impairs blood-brain barrier integrity during ischemic stroke. Nat Med 14:731-737.

Tsirka SE, Rogove AD, Bugge TH, Degen JL, Strickland S (1997) An extracellular proteolytic cascade promotes neuronal degeneration in the mouse hippocampus. J Neurosci 17:543-552.

Volterra A, Meldolesi J (2005) Astrocytes, from brain glue to communication elements: the revolution continues. Nat Rev Neurosci 6:626-640.

Wang YF, Tsirka SE, Strickland S, Stieg PE, Soriano SG, Lipton SA (1998) Tissue plasminogen activator (tPA) increases neuronal damage after focal cerebral ischemia in wild-type and tPA-deficient mice. Nat Med 4:228-231.

Yasuda K, Itakura M, Aoyagi K, Sugaya T, Nagata E, Ihara H, Takahashi M (2011) PKC-dependent inhibition of CA2 +-dependent exocytosis from astrocytes. Glia 59:143-151.

Yepes M, Sandkvist M, Moore EG, Bugge TH, Strickland DK, Lawrence DA (2003) Tissue-type plasminogen activator induces opening of the bloodbrain barrier via the LDL receptor-related protein. J Clin Invest 112:1533-1540.

Zhang C, An J, Strickland DK, Yepes M (2009) The low-density lipoprotein receptor-related protein 1 mediates tissue-type plasminogen activatorinduced microglial activation in the ischemic brain. Am J Pathol 174:586-594.

Zhuo M, Holtzman DM, Li Y, Osaka H, DeMaro J, Jacquin M, Bu G (2000) Role of tissue plasminogen activator receptor LRP in hippocampal longterm potentiation. J Neurosci 20:542-549. 